\title{
CLIP-170 and IQGAP1 Cooperatively Regulate Dendrite Morphology
}

\author{
Lukasz Swiech, ${ }^{1}$ Magdalena Blazejczyk, ${ }^{1,2}$ Malgorzata Urbanska, ${ }^{1}$ Patrycja Pietruszka, ${ }^{1}$ Bjorn R. Dortland, ${ }^{3}$ \\ Anna R. Malik, ${ }^{1}$ Phebe S. Wulf,, ${ }^{3,4}$ Casper C. Hoogenraad, ${ }^{3,4}$ and Jacek Jaworski ${ }^{1}$ \\ ${ }^{1}$ Laboratory of Molecular and Cellular Neurobiology, International Institute of Molecular and Cell Biology, 02-109 Warsaw, Poland, ${ }^{2}$ Nencki Institute of \\ Experimental Biology, 02-093 Warsaw, Poland, ${ }^{3}$ Department of Neuroscience, Erasmus Medical Center, 3000CA Rotterdam, The Netherlands, and \\ ${ }^{4}$ Department of Cell Biology, Faculty of Science, Utrecht University, 3584CH Utrecht, The Netherlands
}

Dendritic arbors are compartments of neurons dedicated to receiving synaptic inputs. Their shape is an outcome of both the intrinsic genetic program and environmental signals. The microtubules and actin cytoskeleton are both crucial for proper dendritic morphology, but how they interact is unclear. The present study demonstrates that microtubule plus-end tracking protein CLIP-170 and actin-binding protein IQGAP1 regulate dendrite morphology of rat neurons by coordinating the interaction between microtubules and the actin cytoskeleton. Moreover, we show that mTOR kinase interacts with CLIP-170 and is needed for efficient formation of a protein complex containing CLIP-170 and IQGAP1. Dynamic microtubules, CLIP-170, and IQGAP1 are required for proper dendritic arbor morphology and PI3K-mTOR-induced increase in dendritic arbor complexity. Moreover, CLIP-170 and IQGAP1 knockdown modulates dendritic arbor growth via regulation of the actin cytoskeleton. We postulate that mTOR controls dendritic arbor morphology by enhancing cross talk between dynamic microtubules and actin through CLIP-170 and IQGAP1.

\section{Introduction}

Dendrites are the major receivers of synaptic inputs in the nervous system. Their development involves several cellular processes, among which both cytoskeletal rearrangement and stabilization play important roles (Urbanska et al., 2008; Jan and Jan, 2010). Microtubules are extremely dynamic structures involved in transport of cellular cargo, maintenance of cell shape, and regulation of cell motility. The dynamic behavior of microtubules is crucial for their function and occurs primarily at the microtubule plus-ends under the control of a heterogeneous family of plus-end tracking proteins (+TIPs) (Schuyler and Pellman, 2001; Akhmanova and Hoogenraad, 2005; Lansbergen and Akhmanova, 2006). In addition to direct regulation of microtu-

Received Dec. 17, 2010; revised Jan. 25, 2011; accepted Feb. 3, 2011.

This research was financed by research grants from the Polish Ministry of Science and Higher Education (2P04A 01530, PBZ-MNil-2/1/2005), a Polish-Norwegian Research Fund grant (PNRF-96-Al-1/07), an FP7 European Union grant (\#223276, "NeuroGSK3"), and an FP7-HealthProt grant (\#229676) to J.J. L.S. was supported by the Polish Cell and Molecular Biology Network and the Centre of Excellence in Molecular Bio-Medicine. M.B. was supported by an FP6 "NovelTune" grant. C.C.H. was supported by the Netherlands Organization for Scientific Research (NWO-ALW/ ECHO), the Netherlands Organization for Health Research and Development (ZonMw-VIDI/TOP), a European Science Foundation European Young Investigator Award, the European Molecular Biology Organization Young Investigators Programme, and a Human Frontier Science Program Career Development Award (HFSP-CDA). We thank Dr. Akhmanova for pSuper-CLIP170sh\#1,2 plasmids, Dr. Sabatini for pRK5-mTOR, and Dr. Sacks for GFP-IQGAP1. We thank Nanda Kajzer, Pawel Krawczyk, Renata Filipek, Marcin Klejman, Iwona Cymerman, Malgorzata Perycz, Monika Dudek, Janusz Debski, and Michal Dadlez for help with the experiments and for their expertise. We thank Drs. Akhmanova, Miaczynska, van Leuven, and Spangler for their useful comments on this work. J.J., L.S., and C.C.H. planned the experiments. L.S., M.B., P.P., M.U., B.R.D., A.R.M., P.S.W., and J.J. performed the experiments and analyzed the data. J.J., L.S., and M.B. prepared the manuscript.

Correspondence should be addressed to Jacek Jaworski, Laboratory of Molecular and Cellular Neurobiology, International Institute of Molecular and Cell Biology, Ks. Trojdena Street 4, 02-109 Warsaw, Poland. E-mail: jaworski@iimcb.gov.pl.

DOI:10.1523/JNEUROSCI.6582-10.2011

Copyright $\odot 2011$ the authors $\quad 0270-6474 / 11 / 314555-14 \$ 15.00 / 0$ bule dynamics, + TIPs are important for intracellular transport and cross talk between microtubules and the actin cytoskeleton (Lansbergen and Akhmanova, 2006; Siegrist and Doe, 2007). In neurons, +TIPs were shown to regulate neuronal survival, migration, and polarization, axon navigation, spine growth and maintenance, and synaptic plasticity [for review, see Jaworski et al. (2008) and Hoogenraad and Bradke (2009)]. However, the role of + TIPs in dendritic arborization has not yet been studied extensively.

Cytoplasmic linker protein of $170 \mathrm{kDa}$ (CLIP-170), a founding member of the +TIP family (Rickard and Kreis, 1990; Perez et al., 1999), is a multifunctional protein whose actions are defined by protein partners interacting on its $\mathrm{N}$ and $\mathrm{C}$ termini (Lansbergen et al., 2004; Galjart, 2005; Steinmetz and Akhmanova, 2008). Interactions between CLIP-170 and the IQ motif-containing GTPase-activating protein 1 (IQGAP1) provide a possible link between microtubules and the actin cytoskeleton by bridging microtubule plus-ends to the cell cortex (Fukata et al., 2002; Watanabe et al., 2004; Kholmanskikh et al., 2006). Notably, + TIP-dependent cross talk between dynamic microtubules and actin is crucial for proper axonal growth and spine maintenance (Geraldo et al., 2008; Hoogenraad and Bradke, 2009; Jaworski et al., 2009) but has not been studied in the context of dendritic arborization.

CLIP-170 has been shown to be phosphorylated at multiple sites in vivo (Rickard and Kreis, 1991), and its phosphorylation is sensitive to rapamycin, an mTOR kinase inhibitor (Choi et al., 2002). Intriguingly, mTOR activity was previously shown to be indispensable for proper dendritic arbor development in hippocampal neurons (Jaworski et al., 2005; Kumar et al., 2005). Altogether, these observations suggest that CLIP-170 may be one 
of the important downstream effectors of mTOR kinase for neuronal morphology and raise the question of how mTOR regulates CLIP-170 activity as needed for proper dendritic arborization.

The present study revealed that dynamic microtubules and CLIP-170 are needed for proper dendritic arbor morphology. Furthermore, both are required for class I phosphoinositide 3-kinase (PI3K)-induced, mTOR-dependent dendritic growth. We provide evidence that mTOR binds CLIP-170 and regulates its interaction with IQGAP1. Consequently, knockdown of IQGAP1 results in simplification of dendritic arborization and inhibition of PI3K-driven growth. Finally, we show that deterioration of dendritic morphology attributable to depletion of either CLIP-170 or IQGAP1 can be prevented by actin stabilization. Thus, our observations strongly suggest a need for mTOR coordinated interaction of dynamic microtubules with the actin cytoskeleton for proper dendritic arbor morphology.

\section{Materials and Methods}

Antibodies and reagents. The following antibodies were obtained from commercial sources: rabbit anti-green fluorescent protein (GFP) (Medical and Biological Laboratories), mouse anti-GFP (Roche Applied Science), mouse anti- $\beta$-galactosidase ( $\beta$-gal) (Promega), rabbit and mouse anti-CLIP-170 (Santa Cruz Biotechnology), rabbit anti-mTOR (Cell Signaling Technology), mouse anti- $\alpha$-tubulin (Sigma), rabbit anti-IQGAP1 (Santa Cruz Biotechnology). Anti-mouse or anti-rabbit Alexa Fluor 488- or 568-conjugated secondary antibodies and horseradish peroxidase (HRP)-conjugated anti-rabbit or anti-mouse secondary antibodies were obtained from Invitrogen and Jackson ImmunoResearch, respectively. Rabbit antiCLIP-170 (\#2360) was previously described (Coquelle et al., 2002). Rapamycin and jasplakinolide were obtained from Calbiochem, and doxycycline and nocodazole were purchased from Sigma.

DNA constructs. The following mammalian expression plasmids have been described previously: pSuper vector (Brummelkamp et al., 2002), pTET-tTS and pSuper $^{\text {TRE }}$ (van de Wetering et al., 2003), $\beta$-actin-GFP, p110CAAX, and myr-Akt (Jaworski et al., 2005), pRK5-myc-mTOR (Kim et al., 2002), EF- $\beta$-gal (Konopka et al., 2005), $\beta$-actin-mRFP (Hoogenraad et al., 2007), GFP-CLIP-170 and GFP-CLIP-170- $\Delta \mathrm{N}$ (Komarova et al., 2002), pEGFPC2-BIO and HA-BirA (de Boer et al., 2003), GFP-IQGAP1 (Ren et al., 2005), and pSuper-CLIP-170sh\#1 and pSuper-CLIP-170sh\#2 (Lansbergen et al., 2004). pSuper-CLIP-170sh\#3 and pSuper-CLIP170sh\#4 sequences were designed against rat CLIP-170 mRNA (NM_031745) targeting the following sequences of the coding sequence (CDS): 1923-1944 (CLIP-170shRNA\#3) and 2271-2289 (CLIP170shRNA\#4). GFP-CLIP-170*3, GFP-CLIP- $170^{\star} 3 \Delta$ N, GFP-CLIP$170^{\star} 3 \Delta \mathrm{C}$, and GFP-CLIP-170*4 GFP-CLIP-170 mutants resistant to sh\#3- and sh\#4-driven RNA interference were generated by mutating the CLIP-170sh\#3 or \#4 target sites, respectively, with following primers: Kruskal-Wallis test).
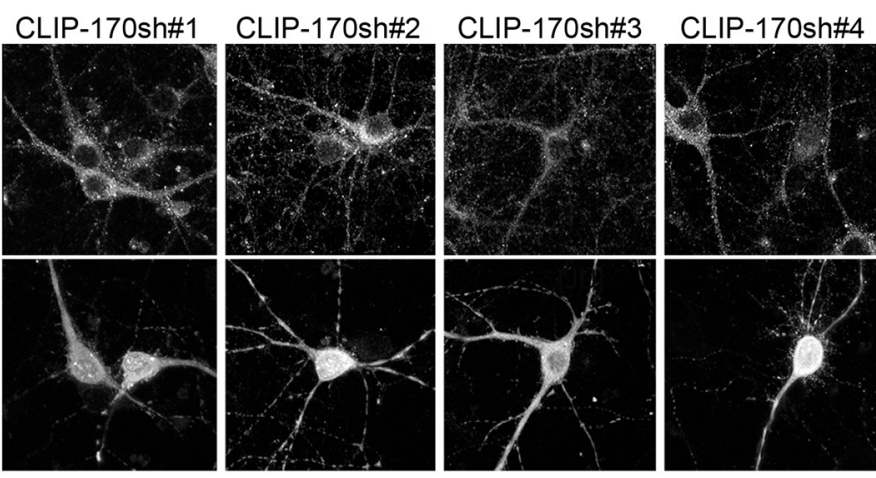

C
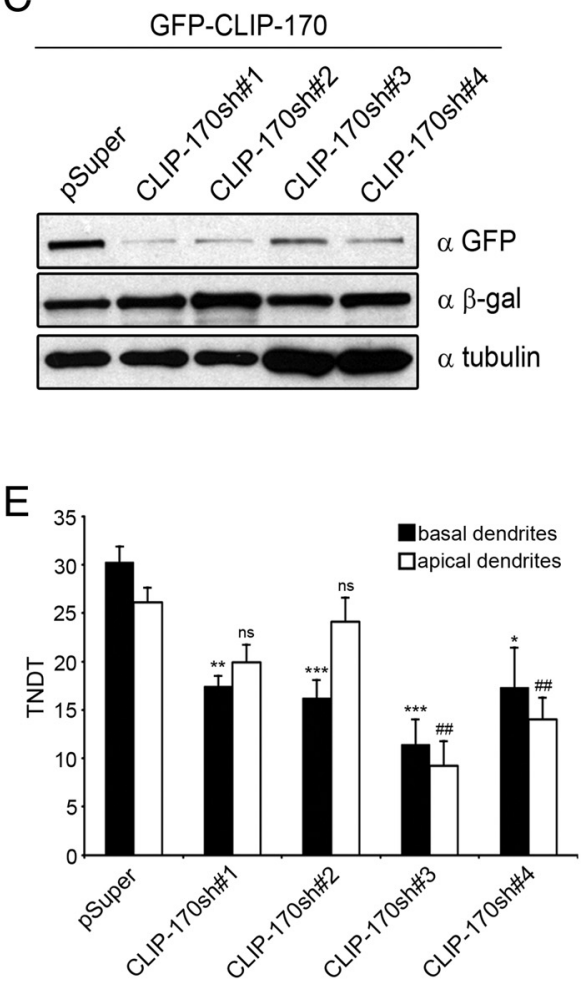

Figure 1. CLIP-170 is needed for proper dendritic arbor morphology of hippocampal neurons. $\boldsymbol{A}$, Representative images of hippocampal neurons cotransfected at DIV7 for $5 \mathrm{~d}$ with control pSuper vector or CLIP-170sh\#1, \#2, \#3, or \#4, stained with antibody against endogenous CLIP-170 and $\beta$-galactosidase for visualization of morphology of transected cells. Scale bar, $10 \mu \mathrm{m}$. $\boldsymbol{B}$, Efficiency of shRNAs was estimated by analysis of average intensity of CLIP-170 immunostaining from transfected cells. Error bars indicate SEM. C, COS7 cells were cotransfected for $2 \mathrm{~d}$ with shRNA constructs targeting rat CLIP-170 (sh\#3 and \#4) or empty vector tive images of neurons in hippocampal slice cultures transfected with pSuper vector or CLIP-170sh\#1 together with GFP, fixed $4 \mathrm{~d}$ after transfection. Scale bar, $100 \mu \mathrm{m}$. $\boldsymbol{E}$, Number of apical and basal dendritic tips of hippocampal neurons in slice cultures after transfection with four different shRNAs (\#1-4) against CLIP-170. Error bars indicate SEM $\left({ }^{* * *} p<0.001,{ }^{* *} p<0.01,{ }^{*} p<0.05\right.$;

CLIP-170*3_F (CAAAGGGATTGGAACTGACTCCGCCGAATTCGCCGAATTCAAAACACAGATAGAGAGACTCAGAC), CLIP-170³_R (GTCTGAGTCTCTCTATCTGTGTTTTGAATTCGGCGAATTCGGCGGAGTCAGTTCCAATCCCTTTG), CLIP-170^4_F (CTGTTAATAAACTGCACCAAAAGGAAGAGCAATTCAACATGTTGTCTTCTGAACTGGAGAAGC), and CLIP-170^4_R (GCTTCTCCAGTTCAGAAGACAACATGTTGAATTGCTCTTCCTTTTGGTGCAGTTTATTAACAG) (boldface indicates nucleotides modified in comparison to wild-type sequence). The GFP-CLIP-170- $\Delta \mathrm{C}$ construct was generated by deletion of the 240 aa part from the $\mathrm{C}$ terminus with BamHI. The fragment of mTOR kinase (501-930 aa) described previously to bind CLIP170 (Choi et al., 2002) was amplified with PCR using pRK5-myc-mTOR as a template and the primers mTOR501 (CGGAATTCATGATCAAGGAGCTGCTGGAGC) and mTOR930 (GTCGTCGACT- 


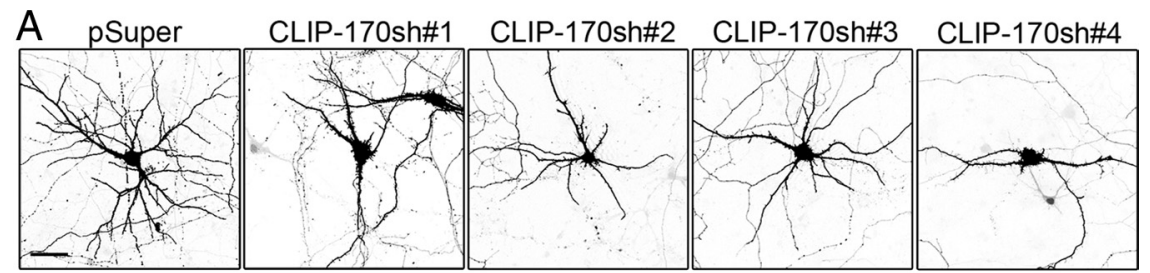

$\mathrm{B}_{35}$

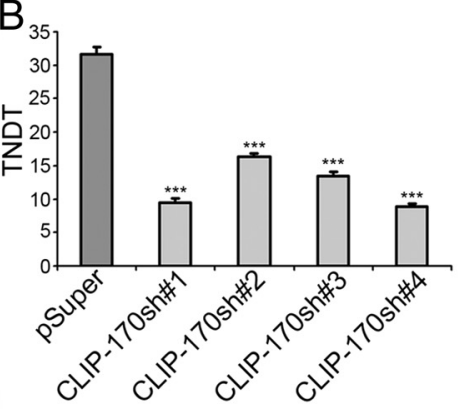

$\mathrm{C}$

$\mathrm{D}$
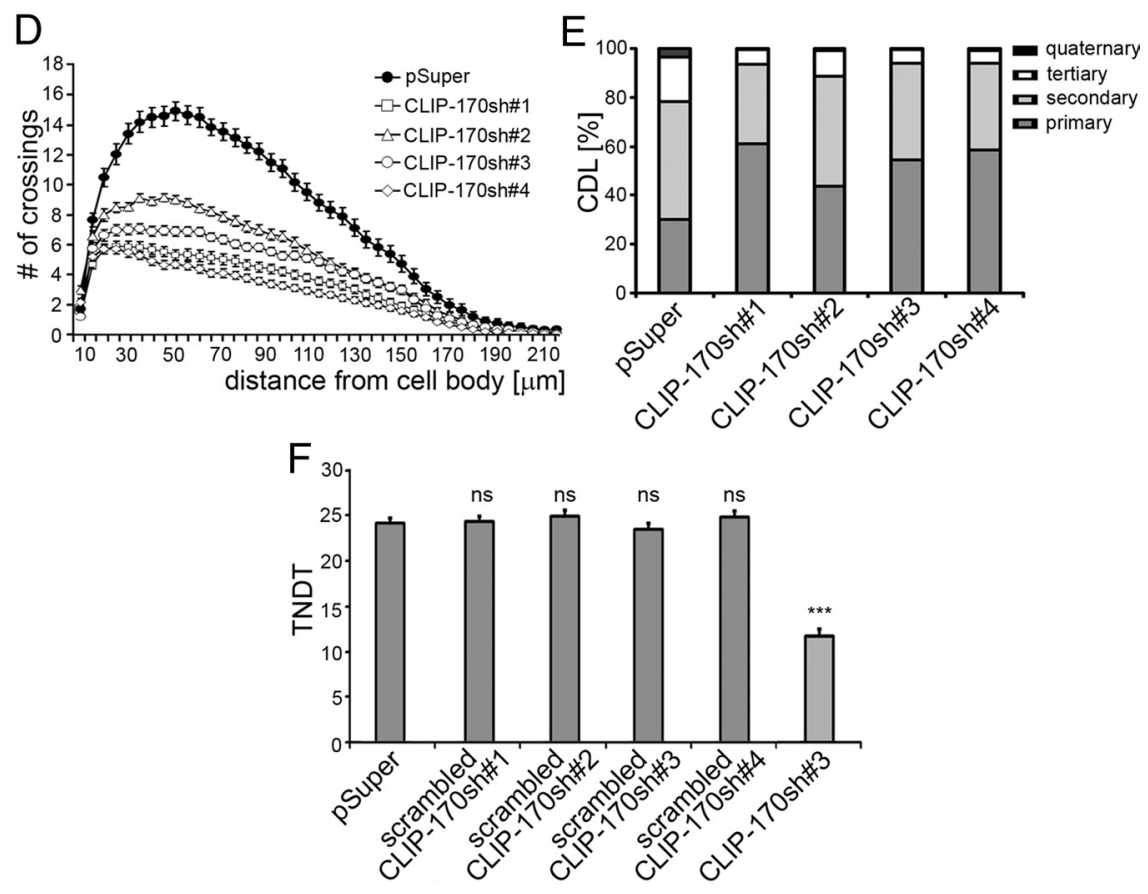

Figure 2. Effects of CLIP-170 depletion on the dendritic arbor morphology of hippocampal neurons in culture.A, Representative images of neurons transfected at DIV7 for $5 \mathrm{~d}$ with pSuper vector or CLIP-170sh\#1, \#2, \#3, or \#4. GFP was cotransfected to identify transfected cells and to visualize neuronal morphology. Scale bar, $20 \mu \mathrm{m} . \boldsymbol{B}-\boldsymbol{E}$, TNDT (B), TDL (C), Sholl analysis (D), and CDL (E) of hippocampal neurons after CLIP-170 knockdown. $\boldsymbol{F}$, TNDT of hippocampal neurons transfected at DIV7 for $5 \mathrm{~d}$ with pSuper or scrambled CLIP-170sh\#1, \#2, \#3, or \#4. Error bars indicate SEM $\left({ }^{* * *} p<0.001\right.$; Kruskal-Wallis test).

CAGCTATAGTCAGAGGAATC), and cloned into EcoRI and SalI restriction sites of an MBP tag encoding pMAL2c vector (New England Biolabs). GFP-IQGAP $1{ }^{*} 2$ GFP-IQGAP1 mutant resistant to IQGAP1sh\#2 was generated by mutating the IQGAP1sh\#2 target site with the following primers: IQGAP1²_F (TTTAGTAACCCTCACAAAACCAGTAATTTATATTTCCATTGGTGAA) and IQGAP1²_R (TTCACCAATGGAAATATAAATTACTGGTTTTGTGAGGGTTACTAAA). To obtain bioGFP-CLIP-170, mTOR, and $\beta$-galactosidase, full-length coding sequences were amplified by PCR using pRK5-mycmTOR, GFP-CLIP-170, and EF- $\beta$-gal as templates and cloned in SalI and NotI restriction sites of pEGFPC2-BIO vector. IQGAP1 shRNA sequences were designed against rat IQGAP1 mRNA (NM_001108489) targeting the following sequences of the coding sequence (CDS): 19431961 (IQGAP1shRNA\#1) and 3872-3890 (IQGAP1shRNA\#2). Oligonucleotides coding these shRNAs were next introduced to pSuper, pSuper ${ }^{\mathrm{TRE}}$, or pSuper ${ }^{\text {GFP }}$ plasmids. pSuper ${ }^{\text {GFP }}$ is a derivative of pEGFP-C1 (Clontech) with the original MCS deleted by BglII/BamHI digestion and a new one reintroduced into the AseI site (kind gift from Dr. Akhmanova, Erasmus Medical Center, Rotterdam, The Netherlands) containing the BamHI/SalI fragment of pSuper with an shRNA cloning cassette cloned into a new MCS, allowing for simultaneous expression of shRNA and GFP. As negative control, in RNAi experiments, pSuper plasmid carrying scrambled shRNA was used in addition to empty plasmid. Scrambled shRNAs were designed based on the original siRNA sequences using on-line GeneScript tool. The following sequences were used: GGCGCAATATGAGACGAAC (scrambledCLIP170sh\#1); CGAGAGAGAGAGAGTTCAT (scrambledCLIP-170sh\#2); GGCTGAGTTTGCTGAGTTAAAG (scrambledCLIP-170sh\#3); TGAGACTAGAAGGTATTGA (scrambledCLIP-170sh\#4); GATCACGTATGCTTCTCGG (scrambledIQGAP1sh\#1); and GGCTAGCGTTCAATCTACA (scrambledIQGAP1sh\#2).

Primary hippocampal and cortical neuron cultures and transfection. Animals used to obtain neurons for tissue cultures were killed according to the protocol approved by a $\mathrm{I}^{\text {st }}$ Ethical Committee in Warsaw, Poland. Primary hippocampal cultures were prepared from embryonic day 19 (E19) rat brains (Banker and Goslin, 1988). Embryos of either sex were used. Cells were plated on coverslips coated with poly-L-lysine $(37.5 \mu \mathrm{g} /$ $\mathrm{ml}$, Sigma $)$ and laminin $(2.5 \mu \mathrm{g} / \mathrm{ml}$, Roche $)$ at a density of 500 cells $/ \mathrm{mm}^{2}$. Hippocampal cultures were grown in Neurobasal medium (Invitrogen) supplemented with B27 (Invitrogen), $0.5 \mathrm{~mm}$ glutamine, $12.5 \mu \mathrm{M}$ glutamate, and penicillin/streptomycin mix (all from Sigma). At day in vitro 8 (DIV8) or DIV15, hippocampal neurons were transfected using Lipofectamine 2000 (Invitrogen) for $5 \mathrm{~d}$ as described by Jaworski et al. (2009). Briefly, for transfection of cells growing in a single well of a 24 -well dish, $1 \mu \mathrm{g}$ of DNA was mixed with $1.67 \mu$ l of Lipofectamine 2000 in $100 \mu \mathrm{l}$ of Neurobasal medium and incubated for $30 \mathrm{~min}$. Fresh culture medium was mixed $1: 1$ with the old one and split into half. The first part was left with the cells, and the second was saved for transfection. Complexes of DNA with Lipofectamine 2000 were then added to the cells and incubated for $4 \mathrm{~h}$ at $37^{\circ} \mathrm{C}$ and $5 \% \mathrm{CO}_{2}$. Afterward, cells were washed twice with Neurobasal and returned to the saved conditioned culture media. When possible, cells were stained for the appropriate epitope tags and GFP to confirm cotransfection. In the RNAi experiments, the shRNA-encoding plasmid and plasmid encoding GFP or mRFP under control of $\beta$-actin promoter ( $\beta$-actin-GFP or $\beta$-actin-mRFP) were cotransfected at a 3:1 ratio. Whenever experiments required doxycycline-controlled expression of shRNA, neurons were cotransfected with pTET-tTS plasmid, pSuper ${ }^{\text {TRE }}$ (empty or carrying shRNA sequence), $\beta$-actin-mRFP, and a GFP-taggedexpressing construct of CLIP-170 at a 1:2:1:1 ratio. Doxycycline was added 1 or $3 \mathrm{~d}$ after transfection to a final concentration of $1 \mu \mathrm{g} / \mathrm{ml}$. Primary cortical cultures were prepared using a similar procedure as hippocampal cultures, with the exception of coating and cell seeding density. Cells were plated on plastic tissue culture dishes $(10 \mathrm{~cm})$ coated with poly-D-lysine $(30 \mu \mathrm{g} / \mathrm{ml}$, 
Sigma) at a density of 1250 cells $/ \mathrm{mm}^{2}$. Rapamycin was added at DIV14 to a final concentration of $100 \mathrm{~nm}$ for $1 \mathrm{~h}$.

Hippocampal slice culture and transfection. Organotypic hippocampal slice cultures were prepared from postnatal day 7 rats as described previously (Stoppini et al., 1991). Slices were grown in an incubator set at $5 \% \mathrm{CO}_{2}, 35^{\circ} \mathrm{C}$, and transfected after $36 \mathrm{~h}$ in vitro using a biolistic method. Hippocampal slices were fixed $4 \mathrm{~d}$ after transfection in $4 \%$ PFA and $4 \%$ sucrose in PBS overnight.

Cell line culture and transfection. RAT-2, HEK293, and COS7 cells were grown in DMEM containing $10 \%$ fetal bovine serum at $37^{\circ} \mathrm{C}$ in $5 \% \mathrm{CO}_{2}$ atmosphere. Cells were transfected using Lipofectamine 2000, according to the manufacturer's protocol.

Immunofluorescence and proximity ligation assay. For immunofluorescent staining of transfected proteins, neurons were fixed with $4 \%$ PFA containing 4\% sucrose in PBS for 10 min at room temperature. For endogenous CLIP-170 protein immunodetection, cells were fixed for $10 \mathrm{~min}$ with ice-cold $100 \%$ methanol with 2 mM EGTA and afterward in $4 \%$ PFA with $4 \%$ sucrose for $10 \mathrm{~min}$ at room temperature. After fixation, cells were washed three times with PBS for $5 \mathrm{~min}$ at room temperature and incubated with primary antibodies in GDB buffer $(0.2 \%$ gelatin, $0.8 \mathrm{M} \mathrm{NaCl}$, $0.5 \%$ Triton $\mathrm{X}-100$, and $30 \mathrm{~mm}$ phosphate buffer, $\mathrm{pH} 7.4$ ) overnight at $4^{\circ} \mathrm{C}$. Cells were then washed three times with PBS for $10 \mathrm{~min}$ at room temperature. Secondary antibodies were applied in GDB for $1 \mathrm{~h}$ at room temperature and underwent three PBS washings, $10 \mathrm{~min}$ each. Secondary antibodies conjugated to AlexaFluor 488 and AlexaFluor 568 were used for double labeling. Generic in situ proximity ligation assay (PLA) was performed according to the manufacturer's (Olink Bioscience) instructions with minor modifications. Briefly, cells after fixation were blocked in Duolink blocking solution for $30 \mathrm{~min}$ at $37^{\circ} \mathrm{C}$ and incubated with primary antibodies in Duolink Antibody diluent solution at $4^{\circ} \mathrm{C}$ overnight. Cells were washed three times in PBS for $10 \mathrm{~min}$ at room temperature. For secondary antibodies conjugated with oligonucleotides, PLA probe anti-mouse MINUS and PLA probe antirabbit PLUS were applied in Duolink antibody diluent solution for $2 \mathrm{~h}$ at $37^{\circ} \mathrm{C}$ and washed three times in PBS for 10 min. Duolink assay was further performed strictly according to the manufacturer's instructions.

Image analysis and quantification. Confocal cell images were obtained with sequential acquisition settings at $1024 \times 1024$ pixel resolution using a Zeiss LSM 5 Exciter microscope. Each image was a $z$-series of images, each averaged twice. The obtained stack was "flattened" into a single image using maximum projection. The confocal settings were kept the same for all scans when fluorescence intensity was compared. Morphometric analysis and quantification were performed using MetaMorph image analysis software (Universal Imaging), and ImageJ with NeuronJ (Meijering et al., 2004) with a modified Sholl plug-in (http://biology. ucsd.edu/labs/ghosh/software). For measurement of total dendrite length, all dendrites of individual neurons were traced. For organotypic cultures, the entire dendritic arbor of each cell was combined using Adobe Photoshop from images acquired at a $20 \times$ objective. Quantification of the number of primary dendrites and dendritic tips and the Sholl analysis were performed on images acquired at a $40 \times$ objective $(0.7$ digital zoom) in dissociated cultures. All nonaxonal protrusions initiating from the cell soma longer than $10 \mu \mathrm{m}$ were defined as primary dendrites. For dendrite tip number, tips of all nonaxonal protrusions longer

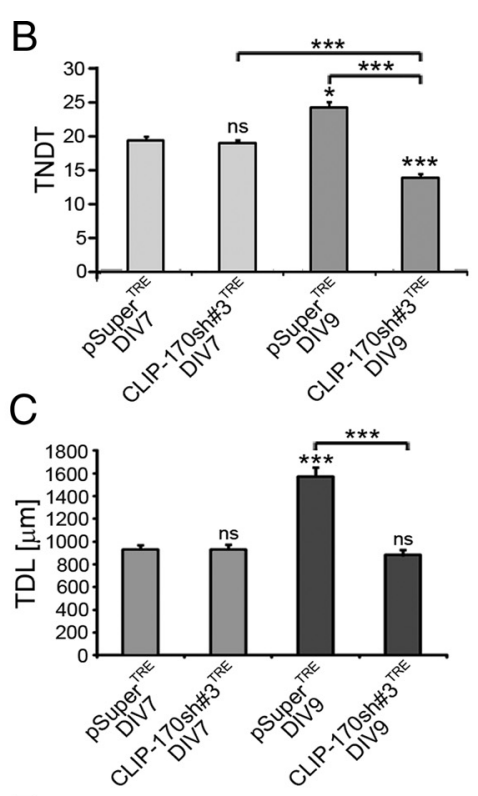

$\mathrm{E}$

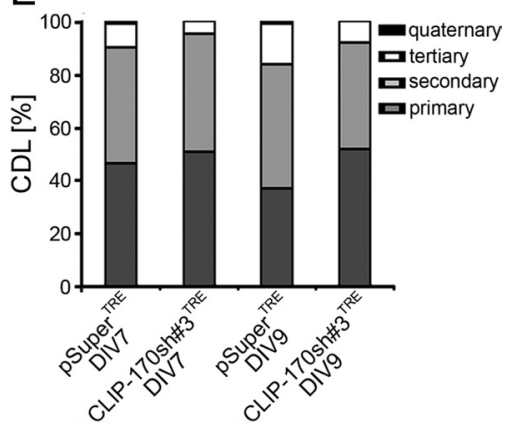

Figure 3. Effects of CLIP-170 depletion on the development of dendritic arbor. Hippocampal neurons at DIV6 were transfected with CLIP-170sh\#3 ${ }^{\text {TRE }}$ or pSuper ${ }^{\text {TRE }}$ and $\beta$-actin-GFP. Doxycycline was added at DIV7 and neurons were fixed immediately or at DIV9. $A$, Schematic representation of a time course of the experiment and representative images of transfected neurons. Scale bar, $20 \mu \mathrm{m}$. $\boldsymbol{B}-\boldsymbol{E}$, TNDT $(\boldsymbol{B})$, TDL $(\boldsymbol{C})$, Sholl analysis $(\boldsymbol{D})$, and CDL $(\boldsymbol{E})$ of hippocampal neurons after CLIP-170 knockdown. Error bars indicate SEM $\left({ }^{* * *} p<0.001,{ }^{*} p<0.05\right.$; Kruskal-Wallis test).

than $10 \mu \mathrm{m}$ were counted. For Sholl analysis, concentric circles with $5 \mu \mathrm{m}$ differences in diameter were automatically drawn around the cell body, and the number of dendrites crossing each circle was counted using ImageJ software with a Sholl plug-in. Data were obtained from two or three independent batches of neurons. Twenty randomly chosen cells were examined for each experimental condition for each culture batch. In the case of organotypic cultures, slices from two independent preparations were used, and a minimum of six slices per experimental condition per preparation were analyzed. For quantification of the effects of shRNAs on levels of endogenous proteins in transfected neurons or RAT-2 cells, cell images were collected using LSM with identical settings across experimental groups and quantified. A similar acquisition regime was used for detection of the PLA-positive signals in either neurons or RAT-2 cells. Both intensity of fluorescence and the number of PLA puncta were quantified with MetaMorph software. Three-dimensional reconstructions of dendrites stained for endogenous CLIP-170 and tubulin were generated from the $Z$-stacks obtained with Zeiss LSM 5 Exciter microscope using Imaris v6.3.1 (Bitplane).

Pull-down and immunoprecipitation. For the pull-down experiment, an mTOR fragment fused to MBP (mTOR501-930MBP) or MBP alone were expressed in the Escherichia coli BL-21 Rosetta strain (Merck). Recombinant proteins were purified from bacterial lysates after induction with $0.4 \mathrm{~mm}$ isopropyl-1-thio- $\beta$-D-galactopyranoside (IPTG, Sigma) on the amylose-Sepharose column according to the manufacturer's protocol (GE Healthcare). Extracts of proteins from adult rat brain were obtained by homogenization of tissue in lysis buffer (40 mM HEPES, pH 7.5, 
A

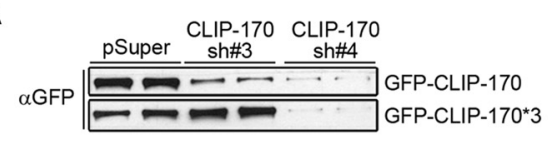

$\mathrm{B}$

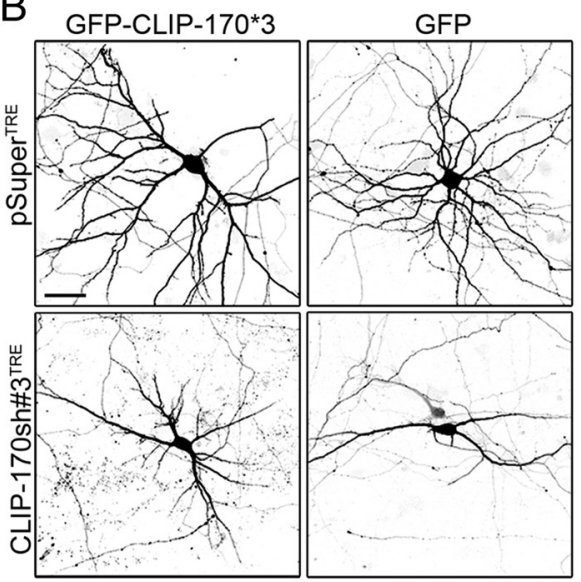

C

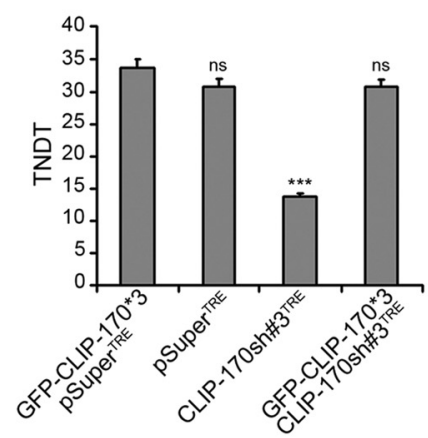

D

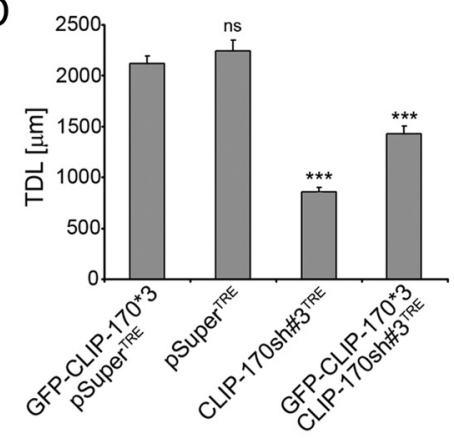

E

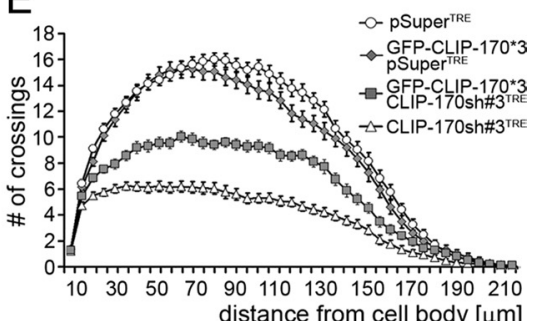

$\mathrm{F}$

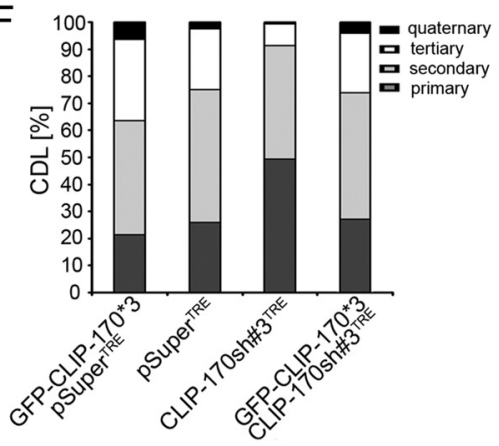

Figure 4. Overexpression ofCLIP-170 in CLIP-170 knockdown cells results in improvement of dendritictreemorphology. $A$, Assessment of GFP-CLIP-170*3 resistance to shRNAs in COS7 cotransfected with wild-type (GFP-CLIP-170) or shRNA\#3-resistant CLIP-170 (GFP-CLIP$170^{*} 3$ ) and pSuper or CLIP-170sh\#3 or \#4 for 48 h. B , Representative images of hippocampal neurons cotransfected at DIV7 with either GFP-CLIP-170*3 or GFP and control pSuper ${ }^{\text {TRE }}$ vector or CLIP-170sh\#3 ${ }^{\text {TRE }}$, treated with doxycycline $(1 \mu \mathrm{g} / \mathrm{ml})$ the next day, and fixed $6 \mathrm{~d}$ after transfection. Neuronal morphology was visualized by cotransfected mRFP. Scale bar, $20 \mu \mathrm{m}$. C $-\boldsymbol{F}$, TNDT (C), TDL (D), Sholl analysis (E), and $\mathrm{CDL}(\boldsymbol{F})$ of hippocampal neurons after transfection. Error bars indicate SEM ( ${ }^{* * *} p<0.001$; Kruskal-Wallis test).

$120 \mathrm{~mm} \mathrm{NaCl}, 0.3 \%$ CHAPS, protease, and phosphatase inhibitors) and put on the prepared resin. After overnight incubation, resins were washed four times with lysis buffer and twice with wash buffer $(50 \mathrm{mM}$ HEPES, $\mathrm{pH} 7.5$, and $150 \mathrm{~mm} \mathrm{NaCl}$ ). For the coimmunoprecipitation experiment, aliquots of adult rat brain lysate were precleared with Sepharose $\mathrm{G}$ (GE Healthcare) at $4^{\circ} \mathrm{C}$ for $30 \mathrm{~min}$ and then immunoprecipitated using rabbit polyclonal antibody against mTOR $(4 \mu \mathrm{g})$ on Sepharose G. Normal rabbit IgG (Sigma) on Sepharose G was used as a negative control $(4 \mu \mathrm{g})$. After overnight incubation at $4^{\circ} \mathrm{C}$, immunoprecipitates were washed extensively similarly to the pull-down experiments. Both pulled-down and immunoprecipitated proteins were dissolved in SDS-PAGE sample buffer and analyzed by Western blotting.

For coimmunoprecipitation of endogenous CLIP-170 and IQGAP1, cultured cortical neurons were harvested in Tris-buffered saline (TBS) and centrifuged at $1000 \times g$ at $4^{\circ} \mathrm{C}$ for $10 \mathrm{~min}$. The supernatant was discarded, and the pellet was homogenized in lysis buffer (10 mM Tris, pH 7.5, $1 \mathrm{~mm} \mathrm{MgCl}_{2}, 0.5 \mathrm{~mm} \mathrm{CaCl}_{2}, 1 \% \mathrm{BSA}, 0.3 \%$ Triton X-100, protease, and phosphatase inhibitors). The lysed cells were incubated on ice at $4^{\circ} \mathrm{C}$ for $1 \mathrm{~h}$ and centrifuged at $14,000 \times g$ at $4^{\circ} \mathrm{C}$ for $30 \mathrm{~min}$ to remove cell debris. Proteins were then immunoprecipitated with Sepharose G (GE Healthcare) conjugated to rabbit anti-IQGAP1 (Santa Cruz Biotechnology) or mouse anti-CLIP-170 (Santa Cruz Biotechnology) $(2 \mu \mathrm{g}$ each). As a negative control, normal mouse or rabbit IgG (Sigma) was used. After overnight incubation at $4^{\circ} \mathrm{C}$, resins were extensively washed four times with lysis buffer and resuspended in SDS-PAGE sample buffer. Samples were analyzed by Western blotting.

F-actin and its binding partners were pulled down from hippocampal protein extracts with use of biotinylated phalloidin (Invitrogen). First, fresh hippocampi from adult rat brain were homogenized in homogenization buffer $(100 \mathrm{~mm}$ phosphate buffer, $\mathrm{pH} 7.2$, containing 2 mм ATP, $2 \mathrm{mM} \mathrm{MgCl}_{2}$, and phosphatase and protease inhibitors). Homogenate was next centrifuged at $800 \times g$ for $15 \mathrm{~min}$ at room temperature, supernatant was divided into two portions, and rapamycin was added to one of them to a final concentration of $100 \mathrm{nM}$ for the duration of the procedure. Biotinylated phalloidin was added to the supernatants to a final concentration of $2 \mathrm{U}$ per sample and incubated for $30 \mathrm{~min}$ at room temperature. At the same time streptavidincoated magnetic beads were prepared by $30 \mathrm{~min}$ incubation in PBS with 5\% BSA. Such pretreated beads were next added to the samples and incubated for $30 \mathrm{~min}$ at room temperature with rotation. The precipitated material was then washed six times with homogenization buffer, resuspended in SDS-PAGE sample buffer, and analyzed by Western blotting.

Production, pull-down, and in vitro phosphorylation of biotinylated proteins. Biotinylated proteins were produced in HEK293 cells cotransfected with bioGFP-mTOR or bioGFP$\beta$-galactosidase together with BirA-encoding plasmid (de Boer et al., 2003) using Lipofectamine 2000. One day after transfection, cells were harvested, washed with TBS, and lysed with lysis buffer $[20 \mathrm{~mm}$ Tris- $\mathrm{HCl}, \mathrm{pH}$ 7.5, $150 \mathrm{~mm} \mathrm{KCl,} 1 \%$ Triton X-100, protease inhibitors (Roche), and phosphatase inhibitor cocktail 1 and 2 (Sigma)]. Cell lysates were centrifuged for $10 \mathrm{~min}$ at $14,000 \times \mathrm{g}$. The supernatant was incubated with streptavidin-coated Dynabeads-280 (Invitrogen) for $1 \mathrm{~h} \mathrm{at} 4^{\circ} \mathrm{C}$. Beads were washed three times with lysis buffer, three times with wash buffer A $(20 \mathrm{~mm}$ Tris, $\mathrm{pH} 7.5,500 \mathrm{~mm} \mathrm{KCl}$, and $0.1 \%$ Triton X-100), and three times with wash buffer B $(20 \mathrm{~mm}$ Tris, pH 7.5, and $150 \mathrm{~mm} \mathrm{KCl).} \mathrm{Neuronal} \mathrm{protein} \mathrm{extracts} \mathrm{were} \mathrm{obtained}$ from DIV14 cultured cortical neurons. Cells were washed with TBS, harvested, and lysed with neuronal lysis buffer (20 mM HEPES, pH 7.5, $150 \mathrm{~mm} \mathrm{NaCl}, 1 \mathrm{~mm}$ EDTA, 0.3\% CHAPS, protease, and phosphatase inhibitors). Lysates were then incubated overnight at $4^{\circ} \mathrm{C}$ with Dynabead resins containing biotinylated proteins produced in HEK293 cells. Afterward, beads were washed four times with neuronal lysis buffer and twice with buffer C (20 mm HEPES, pH 7.5, and $150 \mathrm{~mm} \mathrm{NaCl).} \mathrm{Finally,} \mathrm{beads}$ were resuspended in SDS-PAGE sample buffer and analyzed using Western blotting. In experimental variants involving $\mathrm{mTOR}$ inhibition, neurons were incubated with $100 \mathrm{~nm}$ rapamycin for $1 \mathrm{~h}$ before lysis. The drug was also present in respective cell extracts.

In vitro phosphorylation was performed at $37^{\circ} \mathrm{C}$ in a reaction buffer (450 mm HEPES, pH 7.5, 9 mm EGTA, 0.09\% Tween, and $5 \mu \mathrm{Ci}\left[\gamma_{-}{ }^{32} \mathrm{P}\right]$ ATP) using recombinant, active mTOR kinase fragment (1362-2549 aa; Millipore) and biotinylated GFP-CLIP-170 or GFP- $\beta$-galactosidase bound to Dynabeads. Next, resins were washed several times with washing buffer, resuspended in SDS-PAGE sample buffer, separated by SDSPAGE, and analyzed using PhosphoImager (GE Healthcare).

Statistical analysis. Statistical analysis was performed with GraphPad Prism using the Kruskal-Wallis test with Dunn's multiple-comparisons post hoc test. For comparison of two groups, the Mann-Whitney test was used. 


\section{Results}

Dynamic microtubules contribute to dendritic arbor morphology

Although an important role for + TIPs and their upstream protein kinases has been well described for some aspects of neuronal development and physiology (Jaworski et al., 2008), their participation in acquiring proper dendritic arbor shape has not been investigated. However, inhibition of microtubule dynamics with low doses of nocodazole has been shown to severely impair the formation of primary dendrites (Witte et al., 2008). We used a similar approach to determine whether dynamic microtubules are needed for proper dendritic arbor morphology while dendritic arbor complexity is being established. We also investigated whether nocodazole treatment prevents mTOR-dependent dendritic arbor growth that can be induced by overexpression of constitutively active PI3K-p110CAAX (Jaworski et al., 2005). Hippocampal neurons cultured for $7 \mathrm{~d}$ in vitro were transfected with plasmids encoding $\beta$-gal or p110CAAX and GFP (to visualize morphology of transfected cells) and subsequently treated for $5 \mathrm{~d}$ with 200 nM nocodazole, a dose previously shown to specifically target dynamic microtubules (Jaworski et al., 2009). Nocodazole treatment resulted in a dramatic reduction in the number of dendrites [expressed as total number of dendritic tips (TNDT)] in both $\beta$-gal- $(8.04 \pm 0.56)$ and p110CAAX$(7.59 \pm 0.68)$ transfected cells in comparison to control neurons $(27.94 \pm 1.15$; $p<0.001$, Kruskal-Wallis test). However, TNDT of neurons overexpressing p110CAAX but not treated with the drug increased (37.77 $\pm 1.12 ; p<0.05$, Kruskal-Wallis test), as previously described, suggesting that induced, mTOR-driven dendritic growth strongly depends on dynamic microtubules.

\section{CLIP-170 controls dendritic arbor morphology}

To more specifically address questions regarding role of + TIPs and their regulation in shaping dendritic arbors, we further focused on CLIP-170. We chose this protein because its expression reaches a maximum at the time of intensive dendritogenesis of hippocampal neurons cultured in vitro (Jaworski et al., 2009), and its activity is regulated by mTOR (Choi et al., 2002). To study the function of CLIP-170 in rat neurons, we first designed short-hairpin RNA (shRNA) sequences against rat CLIP-170 mRNA (CLIP-170sh\#3 and \#4). Those sequences introduced in a pSuper vector (Brummelkamp et al., 2002) effectively inhibited CLIP-170 expression (Fig. $1 A-C)$. Sequences with broad species specificity, described earlier (CLIP-170sh\#1 and CLIP-170sh\#2) (Lansbergen et al., 2004), also inhibited CLIP-170 expression (Fig. 1A-C). Moreover, overexpression of CLIP-170sh\#3 and \#4 together with GFP-tagged CLIP-115, another CLIP family member, confirmed the specificity of the shRNAs to CLIP-170 (data not shown). We next tested effects of CLIP170 knockdown for proper dendritic arbor morphology of CA1 neurons in organotypic cultures of hippocampus. As shown in Figure 1, $D$ and $E$, biolistic transfection of pSuper plasmids encoding all four CLIP-170 shRNAs to CA1 pyramidal neurons at DIV1 for $5 \mathrm{~d}$ induced dendritic arbor simplification manifested as a significant reduction in TNDT of basal dendrites.

To better understand role of CLIP-170 in shaping dendritic arbor, we took an advantage of in vitro cultures of dissociated hippocampal neurons, which allow for easy genetic and pharmacological manipulations. We first confirmed the morphological effects of CLIP-170 knockdown 5 d after transfection of DIV7 hippocampal neurons with CLIP-170sh plasmids (Fig. 2 A). Indeed, overexpression of each of the shRNAs against CLIP-170, but not corresponding scrambled controls, significantly decreased TNDT by at least 50\% (Fig. $2 B, F$ ). Notably, overexpression of a CLIP-170 mutant lacking the N-terminal part of the protein, which cannot bind microtubules (GFP-CLIP-170 $\Delta \mathrm{N}$ ), also resulted in the $\sim 20 \%$ decrease in TNDT (data not shown). Analysis of total dendritic length (TDL) also revealed deterioration of dendrites induced by CLIP-170 knockdown (Fig. 2C). 


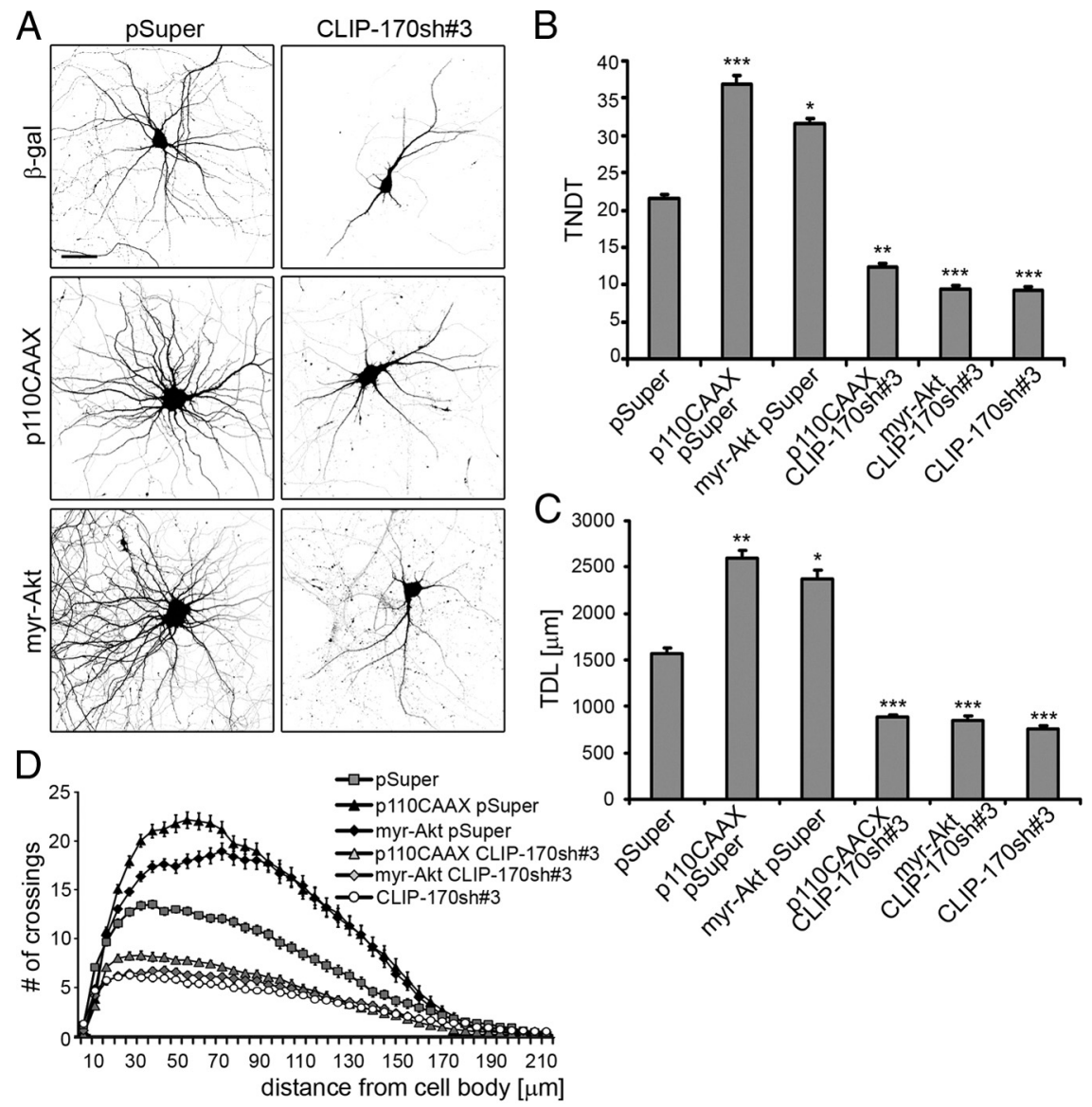

Figure 6. CLIP-170 is necessary for PI3K- and Akt-induced increases in dendritic arbor complexity. $\boldsymbol{A}$, Representative images of hippocampal neurons cotransfected at DIV7 with CLIP-170sh\#3 orpSuper control vector together with $\beta$-galactosidase $(\beta$-gal), p110CAAX, or myr-Akt and fixed 5 dafter transfection. GFP was used for cell morphology visualization. Scale bar, $20 \mu \mathrm{m}$. $\boldsymbol{B}-\boldsymbol{D}, \mathrm{TNDT}(\boldsymbol{B})$, TDL (C), and Sholl analysis $(\boldsymbol{D})$ of hippocampal neurons after transfection. Error bars indicate SEM $\left({ }^{* * *} p<0.001,{ }^{* *} p<0.01,{ }^{*} p<0.05\right.$; Kruskal-Wallis test).

TNDT and TDL are quantitative measures of absolute changes in dendrite number and overall dendritic tree size. However, they do not provide any information about changes in the shape or complexity of the dendritic arbor and the overall pattern of arborization. To describe the observed changes in neuronal morphology more globally, we used two additional types of measurements: a Sholl analysis (Sholl, 1953) and an analysis of the contribution of dendrites of different order to TDL (CDL). The Sholl analysis revealed that in neurons with CLIP-170 knockdown, the most intensive dendritic branching occurred much closer to the cell soma, and the number of dendrites at almost all measured distances was lower than in control cells (Fig. 2D). CDL analysis indicated that higher-order dendrites were the most affected by CLIP-170 knockdown. shRNA transfection increased the participation of primary dendrites and concurrently decreased the contribution of higher-order branches (Fig. 2E).

A reduction of number of dendrites may result from either their decreased growth or accelerated retraction. Thus, we decided to distinguish these two situations, taking advantage of doxycyclineinduced expression of shRNAs (Hoogenraad et al., 2005). We assumed that in the first case, dendritic arbors of cells fixed immediately and $2 \mathrm{~d}$ after stimulation of CLIP-170sh expression should be comparable, while dendritic trees of control neurons would show increased complexity. On the other hand, if CLIP-170 knockdown accelerates dendritic retractions, dendritic trees of neurons fixed at later time points should be dramatically reduced in comparison to early fixed ones. CLIP-170sh\#3 was first cloned into pSuper ${ }^{\mathrm{TRE}}$ plasmid, which guarantees doxycycline-induced shRNA expression and cotransfected into DIV6 neurons with plasmid encoding tetracycline transrepressor (tTS). pSuper ${ }^{\mathrm{TRE}}$ was used as negative control. Doxycycline was added $24 \mathrm{~h}$ after transfection, and cells were fixed either immediately afterward or $48 \mathrm{~h}$ later (Fig. 3A). As shown in Figure 3, during 2 d, TNDT, TDL, and dendritic arbor complexity of control cells increased significantly. Also, contribution of higher-order dendrites to the total length rose. Overexpression of CLIP-170sh\#3 for $2 \mathrm{~d}$, however inhibited these changes (Fig. $3 A-E$ ). However, when dendritic arbors of DIV9 neurons transfected with CLIP-170sh\#3 ${ }^{\text {TRE }}$ were compared with those of DIV7 cells (control and expressing sh\#3), we observed no differences in TDL and Sholl analysis and only small changes in TNDT and CDL (Fig. $3 B-E)$. Analysis of CDL revealed additionally slight leakiness of doxycycline-induced shRNA expression system, since in neurons after CLIP-170sh\#3 ${ }^{\text {TRE }}$ transfection at DIV6 small changes have been already visible at DIV7, although doxycycline was not added. Altogether, those results suggest that CLIP-170 is indispensable for acquiring proper dendritic arbor morphology of hippocampal neurons in various in vitro preparations.

\section{Full-length CLIP-170 is crucial for proper dendritic arborization}

To confirm the specificity of the observed effects of CLIP-170 knockdown on dendritic arbor morphology, we "rescued" the CLIP-170 knockdown phenotype overexpressing GFP-tagged CLIP-170*3. CLIP-170*3 is not recognized by CLIP-170sh\#3 because of point mutations in the targeting sequence, which was confirmed in COS7 cells transfected with CLIP-170sh\#3 and CLIP-170sh\#4 (Fig. 4A). We tested whether transfection of CLIP $-170 * 3$ reverses the deleterious effects of CLIP-170 knockdown in neurons. To allow production of CLIP $-170^{\star} 3$ before knockdown of endogenous protein, neurons were transfected at DIV7 with pTET-tTS, pSuper ${ }^{\text {TRE }}$ or CLIP-170sh $\# 3^{\text {TRE }}$ and CLIP-170*3 and next treated with doxycycline $24 \mathrm{~h}$ after transfection until fixed $5 \mathrm{~d}$ later (Fig. $4 B$ ). We decided to use the inducible system to avoid a potential situation that knockdown of CLIP-170 would precede production of CLIP-170*3, because experiments described above clearly showed that even small decrease of CLIP-170 level due to leakiness of the Tet system was sufficient to initiate morphological changes. Overexpression of CLIP-170*3 fully reversed the negative effects of CLIP-170sh\#3 ${ }^{\text {TRE }}$ on TNDT and CDL to the levels of control neurons transfected with CLIP-170*3 and pSuper ${ }^{\text {TRE }}$ or pSuper ${ }^{\text {TRE }}$ alone (Fig. $4 C, F$ ). CLIP-170*3 overexpression also partially rescued the decrease in TDL and the dendritic field shape changes caused by CLIP-170sh\#3 ${ }^{\text {TRE }}$ (Fig. 4D) and shifted the Sholl plot rightward to the control position but did not provide a complete upward shift (Fig. $4 E$ ). Comparable results were 
obtained for CLIP-170sh\#4 $4^{\text {TRE }}$ and its rescue construct CLIP- $170 * 4$ (data not shown).

To understand the molecular basis for CLIP-170 engagement in dendrite morphogenesis, we investigated which particular functional domains of CLIP-170 are needed to support dendritic arbor complexity. We constructed deletion mutants of CLIP-170*3 lacking either the $\mathrm{N}$ or $\mathrm{C}$ terminus (Fig. 5A). The silent mutation in the shRNA\#3-recognized sequence did not alter the expected cellular distribution of these proteins overexpressed in RAT-2 cells (Pierre et al., 1994; Komarova et al., 2002) (data not shown). Additionally knockdown of endogenous CLIP-170 did not influence expected distribution of the mutants (data not shown).

Plasmids encoding CLIP-170*3 mutants were transfected to hippocampal neurons at DIV7 for $6 \mathrm{~d}$, together with CLIP-170sh\#3 ${ }^{\text {TRE }}$ and pTET-tTS. Expression of shRNA was induced by the addition of doxycycline $24 \mathrm{~h}$ after transfection. Although overexpression of full-length GFP-tagged CLIP-170*3 fully reversed the negative effects of CLIP-170sh\#3 on TNDT to control levels (Fig. 4C), the use of CLIP-170*3 deletion mutants did not provide full restoration (Fig. $5 B, C$ ). Both mutants were also less efficient than full-length CLIP-170*3 at rescuing other measured parameters of dendritic morphology (Fig. 5D-F). Thus, the results from these rescue experiments using CLIP-170 deletion mutants provide evidence that intact, full-length CLIP-170 is required for proper dendritic arbor morphology, suggesting the importance of its protein-protein interactions at both the $\mathrm{N}$ and $\mathrm{C}$ termini.

CLIP-170 is required for dendritic growth after activation of PI3K signaling pathway

We previously showed that the PI3K-AktmTOR signaling pathway is important for proper dendritic arbor development in hippocampal neurons (Jaworski et al., 2005), and we revealed that dynamic microtubules are needed for PI3K-induced dendritic arbor growth. Unknown is whether CLIP-170 is important for PI3Kinduced dendritic arbor growth. If so, then knockdown of CLIP-170 should prevent dendritic growth induced by overexpression of p110CAAX or constitutively active Akt (myr-Akt). To test this hypothesis, we cotransfected DIV7 neurons with either p110CAAX or myr-Akt together with CLIP-170sh\#3 or pSuper for $5 \mathrm{~d}$. Overexpression of the constitutively active kinases alone led to a significant increase in TNDT and TDL and a rightward and upward shift of the Scholl plot (Fig. 6A-D). CLIP-170sh\#3 cotransfection completely
A

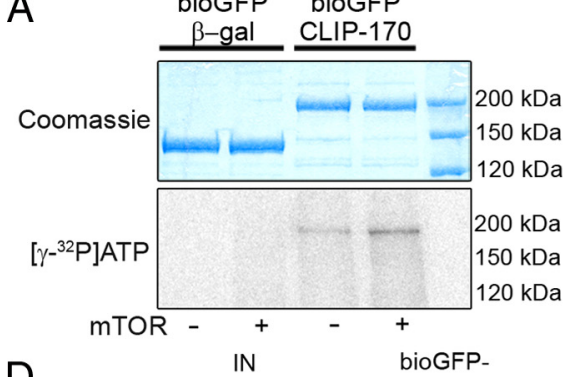

D

$E$

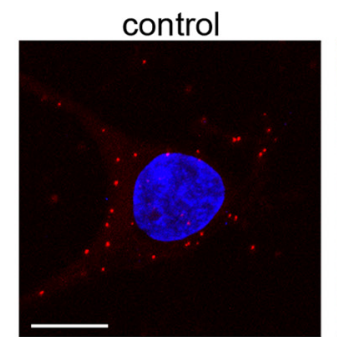

F
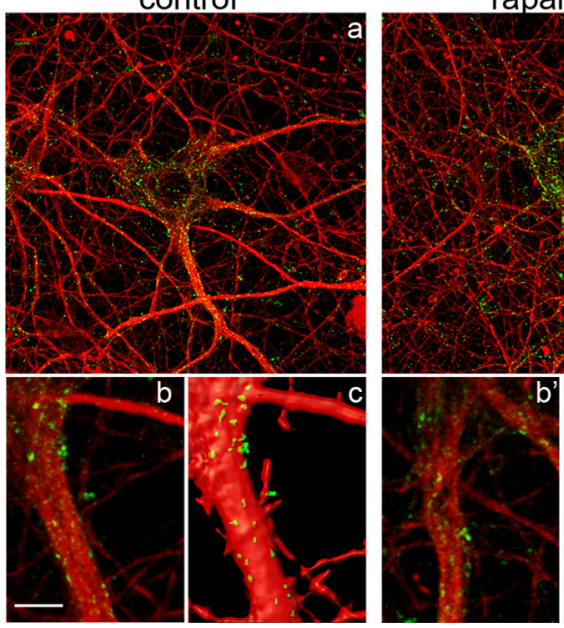

B

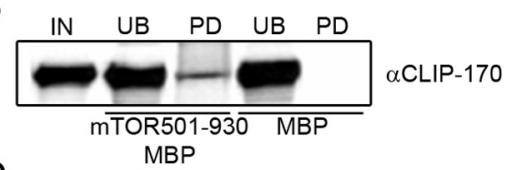

C

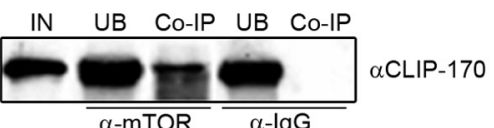

bioGFP
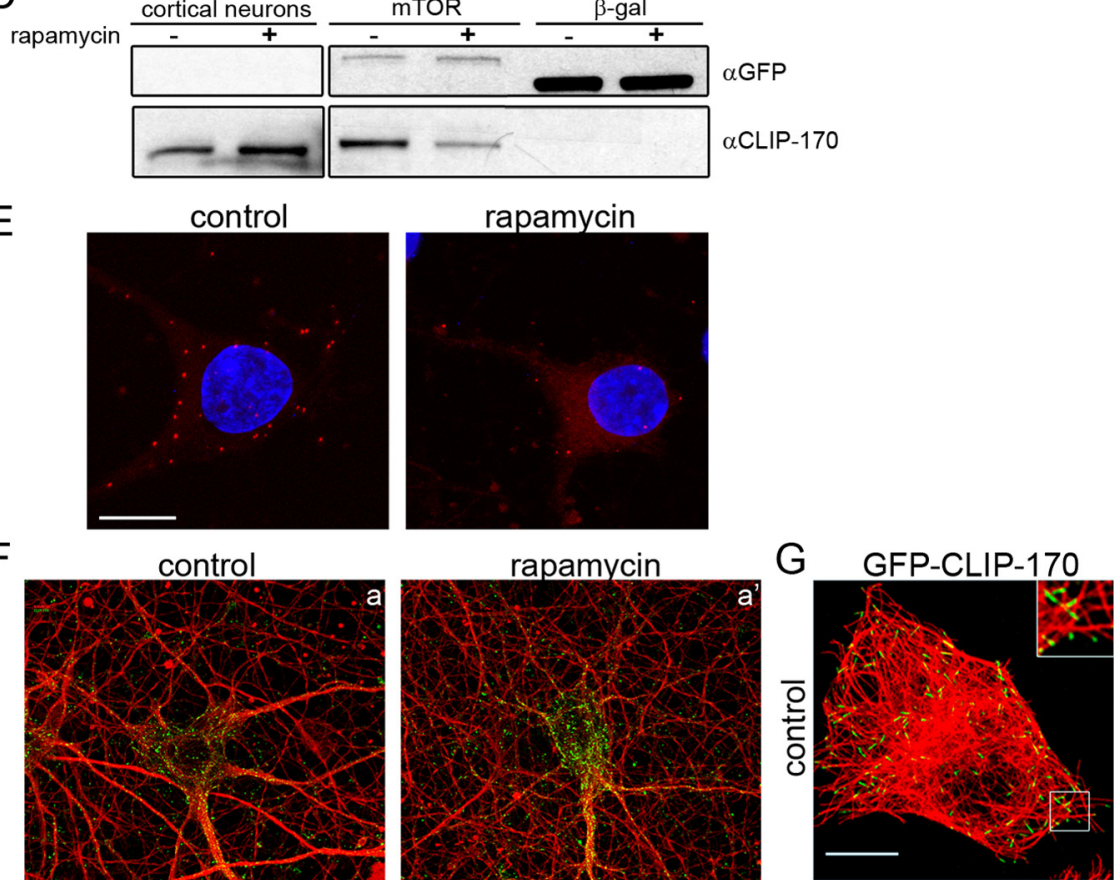

Figure 7. CLIP-170 protein interactions are regulated in an mTOR-activity-dependent manner. $\boldsymbol{A}$, Recombinant active mTOR fragment increases incorporation of $\left[\gamma^{-32}\right.$ P]ATP to bioGFP-CLIP-170 in vitro. $\boldsymbol{B}$, Results of pull-down of CLIP-170 with MBP-fused mTOR fragment (501-930 aa) from rat brain protein lysate. Fractions: IN, input; UB, unbound; PD, pull-down. C, Coimmunoprecipitation of CLIP-170 and mTOR from rat brain protein lysate. Fractions: IN, input; UB, unbound; Co-IP, coimmunoprecipitation. D, Pull-down of CLIP-170 and mTOR from lysates of cortical neurons with overexpressed in vivo biotinylated GFP-mTOR (bioGFPmTOR) and GFP- $\beta$-galactosidase (bioGFP- $\beta$-gal), bound to streptavidin resins. Where indicated, neurons were treated with 100 $\mathrm{nm}$ rapamycin $1 \mathrm{~h}$ before lysis. $\boldsymbol{E}$, Representative image of proximity ligation assay detection of protein complexes containing CLIP-170 and mTOR in hippocampal neurons incubated with $100 \mathrm{~nm}$ rapamycin or vehicle for $1 \mathrm{~h}$. Red signal represents positive PLA reaction, and DAPI staining (blue) highlights the nucleus. Scale bar, $10 \mu \mathrm{m}$. $\boldsymbol{F}$, Representative images of hippocampal neurons in culture at DIV10, stained with antibodies against CLIP-170 (green) and $\alpha$-tubulin (red). CLIP-170 colocalizes with $\alpha$-tubulin in dendrites and cell body $(\boldsymbol{a}, \boldsymbol{b})$. After $1 \mathrm{~h}$ incubation with rapamycin (100 nM), CLIP-170 was still present at the microtubules $\left(\boldsymbol{a}^{\prime}, \boldsymbol{b}^{\prime}\right)$. 3 D reconstruction of CLIP-170 clusters in dendrites $\left(\boldsymbol{c}, \boldsymbol{c}^{\prime}\right)$. Scale bar, $5 \mu \mathrm{m}$. G, HeLa cells transfected with GFP-CLIP-170 (green) and treated with rapamycin (100 nm) for $1 \mathrm{~h}$ ( $\alpha$-tubulin immunostaining in red). Scale bar, $20 \mu \mathrm{m}$.

blocked the effect of overexpression of p110CAAX and myr-Akt (Fig. 6A-D). These data indicate that CLIP-170 is needed for PI3K-induced dendrite arborization.

Active mTOR exists in protein complex with CLIP-170

Dynamic microtubules and CLIP-170 are needed for mTORcontrolled dendritic growth, and we hypothesized that, similar to 
non-neuronal cells, CLIP-170 might also be regulated by mTOR in neurons and that this regulation is crucial for dendrite growth. Previous work has shown that the microtubule binding ability of CLIPs depends on their phosphorylation status (Rickard and Kreis, 1991; Hoogenraad et al., 2000; Choi et al., 2002). Choi et al. (2002) showed that application of the mTOR inhibitor rapamycin led to CLIP-170 dephosphorylation and depletion from microtubule plus-ends (Choi et al., 2002). We tested whether (1) mTOR directly phosphorylates CLIP-170, (2) mTOR and CLIP170 coexist in protein complex in neurons, and (3) mTOR regulates the interactions of CLIP-170 with interacting proteins.

We first showed that a recombinant active fragment of mTOR kinase (amino acids 1360-2549) increased phosphorylation of biotinylated GFP-CLIP-170 (bioGFP-CLIP-170; 6.9-fold increase in comparison to control) but not GFP- $\beta$-galactosidase (bioGFP- $\beta$-gal) purified from HEK293 cells (Fig. 7A). We next tested whether CLIP-170 from rat brain extracts interacts with $\mathrm{mTOR}^{501-930}$, a protein fragment previously identified by a yeast two-hybrid screen to interact with CLIP-170 (Choi et al., 2002). In fact, brain-derived CLIP-170 was pulled down by mTOR $^{501-930}$ tagged with maltose-binding protein $(\mathrm{MBP})$ but not by MBP alone (Fig. $7 B$ ). This result was further confirmed by coimmunoprecipitation of endogenous CLIP-170 with mTOR from rat brain extracts (Fig. 7C). Thus, mTOR and CLIP-170 likely coexist in the same protein complex in the brain, and CLIP-170 can serve as a substrate for mTOR.

We next sought to determine whether formation of the CLIP$170-$ mTOR protein complex depends on mTOR activity. We used in vitro cultures of cortical neurons to allow simple pharmacological manipulations and provide sufficient material for biochemical analysis. We first confirmed the CLIP-170-mTOR interaction in cortical neuron lysates using a biotin-streptavidin pull-down method (de Boer et al., 2003). Biotinylated GFPmTOR (bioGFP-mTOR) or GFP- $\beta$ GAL was produced in HEK293 cells and incubated with protein extracts from DIV14 cortical neurons. When mTOR was not inhibited, endogenous CLIP-170 bound to bioGFP-mTOR, but not to bioGFP- $\beta$-gal (Fig. 7D). However, $1 \mathrm{~h}$ treatment of neurons with $100 \mathrm{~nm}$ rapamycin and the presence of the drug in cell extracts decreased CLIP-170 binding to bioGFP-mTOR resin (Fig. 7D). We further confirmed this observation using an independent method, a PLA, which allows the study of protein interactions in individual cells in situ. PLA probes are secondary antibodies labeled with oligonucleotides, which hybridize and form a template for rollingcircle amplification (RCA), only when proteins of interest are in close proximity $(<40 \mathrm{~nm})$. RCA product can be next visualized by a fluorescent in situ hybridization. The positive signals of detected pairs of PLA probes are visualized as fluorescent spots, which can be quantified. The PLA-positive signal was clearly visible in hippocampal neurons under basal conditions but was severely reduced upon the addition of $100 \mathrm{~nm}$ rapamycin for $1 \mathrm{~h}$ (control, $100 \pm 11.37 \%$; rapamycin, $41.18 \pm 6.57 \%$; $p<0.001$; Mann-Whitney test) (Fig. 7E). These data show that the CLIP170 interaction with mTOR depends on mTOR kinase activity.

It was previously shown that rapamycin treatment blocked an ability of CLIP-170 to bind microtubules in HeLa cells (Choi et al., 2002). To test this effect in neuronal cells, we checked distribution of endogenous CLIP-170 and tubulin in hippocampal neurons treated for $1 \mathrm{~h}$ with $100 \mathrm{~nm}$ rapamycin and did not detect any striking changes in CLIP-170 immunostaining pattern (Fig. $7 F$ ). Regardless of mTOR inhibition, similar distribution of comet-like structures of CLIP-170 were observed in cell bodies, axons, and dendrites. This lack of an effect of rapamycin on
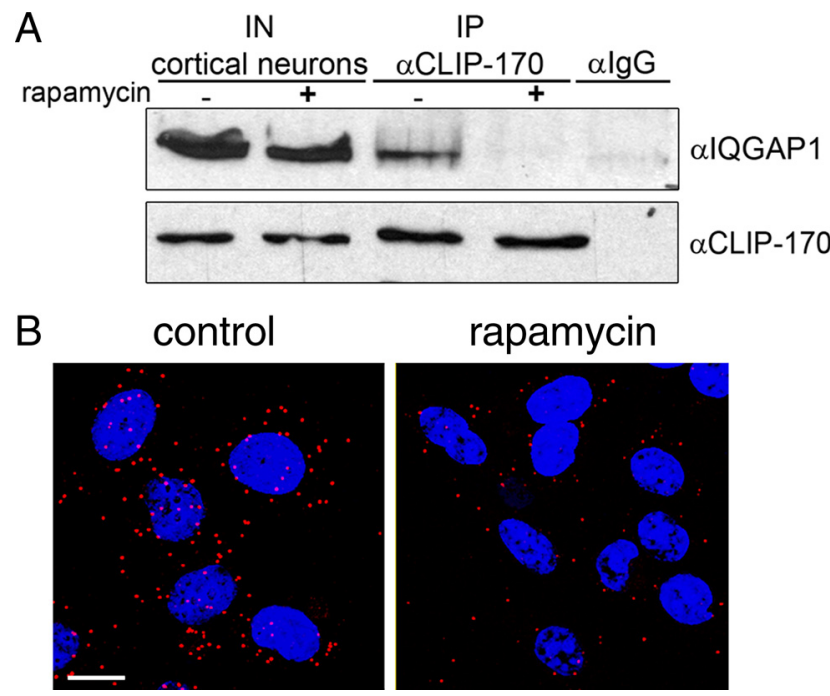

Figure 8. IQGAP1 and CLIP-170 interaction is regulated by mTOR kinase activity. $\boldsymbol{A}$, Coimmunoprecipitation of CLIP-170 and IQGAP1 from lysates of cortical neurons. Where indicated, cells were treated with $100 \mathrm{~nm}$ rapamycin before lysis. $\boldsymbol{B}$, Representative results of proximity ligation assay detection of protein complexes containing CLIP-170 and IQGAP1 in RAT-2 cell line. One hundred nanomolar rapamycin was added $1 \mathrm{~h}$ before fixation. Red signal represents PLA-positive reaction, and DAPI staining (blue) highlights the nucleus. Scale bar, $20 \mu \mathrm{m}$.

CLIP-170 distribution was not unique for neurons, since incubation of HeLa cells transfected with GFP-CLIP-170 with $100 \mathrm{nM}$ rapamycin also did not cause detachment of GFP-CLIP-170 from the microtubule plus-ends (Fig. $7 G$ ). Thus, we concluded that control of CLIP-170 localization to a microtubule plus-end is not a major outcome of mTOR action toward CLIP-170.

\section{Active mTOR regulates the CLIP-170-IQGAP1 interaction in neurons}

We next determined whether mTOR activity can control other CLIP-170 interactions. An actin-binding protein, IQGAP1 is a known interacting partner of CLIP-170 (Fukata et al., 2002). Coimmunoprecipitation experiments showed that these two proteins interact also in neurons (Fig. 8A). Thus we tested whether this particular interaction of CLIP-170 depends on mTOR and indeed revealed that coimmunoprecipitation of IQGAP1 with CLIP-170 was much less efficient when cells were treated with rapamycin ( $42 \%$ of control $\pm 7.2 \%$; $p<0.01$, Mann-Whitney test). This observation was further corroborated by a strong decrease in IQGAP1-CLIP-170 PLA signal in RAT-2 fibroblasts when treated with $100 \mathrm{~nm}$ rapamycin (control, $100 \pm 11.37 \%$; rapamycin, $47.36 \pm 3.1 \%$; $p<0.001$, Mann-Whitney test) (Fig. $8 \mathrm{~B})$. Altogether, these results support our hypothesis that mTOR activity regulates the interaction of CLIP-170 with other factors, including mTOR itself and IQGAP1.

\section{IQGAP1 and CLIP-170 cooperate with each other for proper} dendritic arbor morphology

The evidence provided above implicates CLIP-170 in shaping dendritic arborization and suggests IQGAP1 as its interacting partner. We therefore determined whether knockdown of IQGAP1 simplifies dendritic arborization. First, we designed shRNAs against rat IQGAP1 mRNA, cloned them into pSuper ${ }^{\text {GFP }}$, and confirmed their efficacy by transfecting RAT-2 fibroblasts and probing for levels of endogenous IQGAP1 protein (Fig. 9A,B). The same shRNAs in pSuper plasmid were then introduced to DIV7 neurons. Transfection of both IQGAP1 shRNA signifi- 
cantly decreased TNDT compared with control cells (Fig. 9C,D). On the other hand, when neurons were transfected with matching scrambled shRNAs, we did not note TNDT decrease (Fig. 9H). Surprisingly, TDL was not affected and dendritic arbor complexity decreased only slightly after IQGAP1 depletion (Fig. $9 E, F)$. There was also a decrease in contribution of higher-order branches to TDL (Fig. 9G). Thus, the strongest effect of IQGAP1 knockdown was the decrease in TNDT, and in the following experiments, we focused only on this parameter. It is worth noting, however, that even in this case, observed difference was smaller than with CLIP-170 knockdown.

To further confirm specificity of phenotypic effects of IQGAP1 depletion, we performed the rescue experiment using IQGAP $1{ }^{\star} 2$, an IQGAP1 mutant resistant to IQGAP1sh\#2 (Fig. 10A). DIV7 neurons were cotransfected with GFP-IQGAP1*2 and IQGAP1sh $\# 2^{\text {TRE }}$ and treated with doxycycline $1 \mathrm{~d}$ later. In additional control variants, cells were cotransfected with pEGFP or GFP-IQGAP1 ${ }^{\star} 2$ and pSuper ${ }^{\text {TRE }}$. Doxycycline was added the next day and neurons were fixed at DIV13 (Fig. 10B). As shown in Figure 10C, transfection of GFPIQGAP $^{*} 2$ prevented the decrease in TNDT caused by IQGAP1 depletion. Interestingly, overexpression of GFP-IQGAP1 ${ }^{\star} 2$ alone in neurons resulted in TNDT decrease, strongly suggesting that precise regulation of IQGAP1 level is needed for a proper dendritic arbor morphology.

Since interaction of IQGAP1 and CLIP170 is regulated by mTOR, we next tested whether IQGAP1 similarly to CLIP-170 is required for PI3K-induced, mTORdependent dendritogenesis. Analogous to experiments described above, we cotransfected DIV7 neurons with p110CAAX and IQGAP1sh\#2 or pSuper. Cotransfection of IQGAP1sh\#2 prevented the permissive effect of p110CAAX overexpression on neuronal morphology (Fig. 11 $A, B$ ). Thus, both CLIP-170 and IQGAP1 are needed for PI3K-induced dendritic arborization. Now the question arises of whether the interplay between these two proteins is required for proper dendritic arbor morphology. To answer it, we performed reciprocal rescue experiments. As shown in Figure 11 when GFP-CLIP-170 was transfected together with IQGAP1sh\#2, TNDT was comparable to one of control (Fig. 11C,D). Furthermore, the rescue experiment of CLIP-170 knockdown by GFP-IQGAP1 overexpression led also to significant improvement of TNDT (Fig. 11E,F) despite deterioration in the dendritic tree morphology caused by overexpression of IQGAP1 alone (Fig. 10C). These results suggest interdependence of IQGAP1 and CLIP-170 in shaping dendritic arbor. Wallis test).
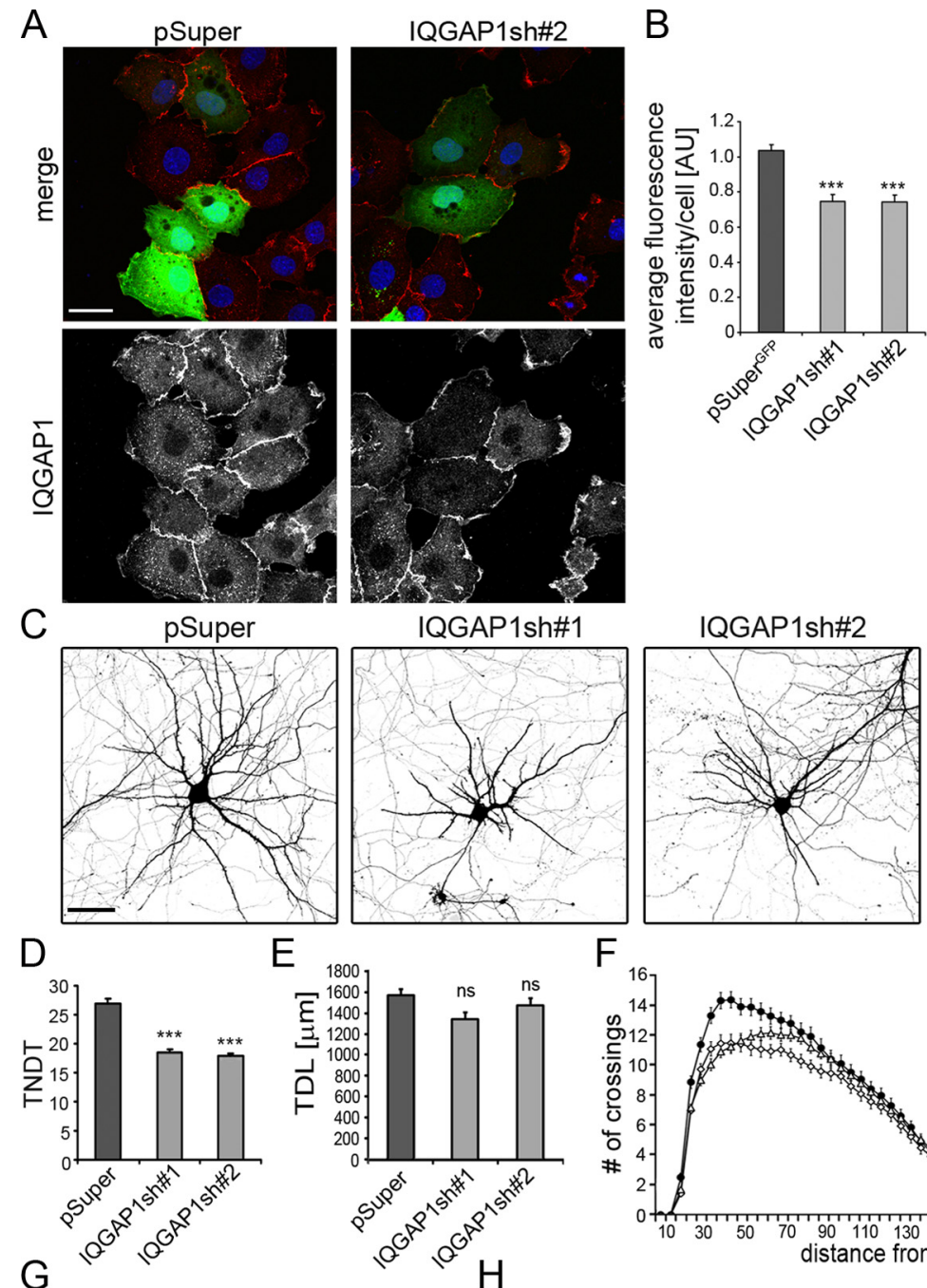

$F_{10}$
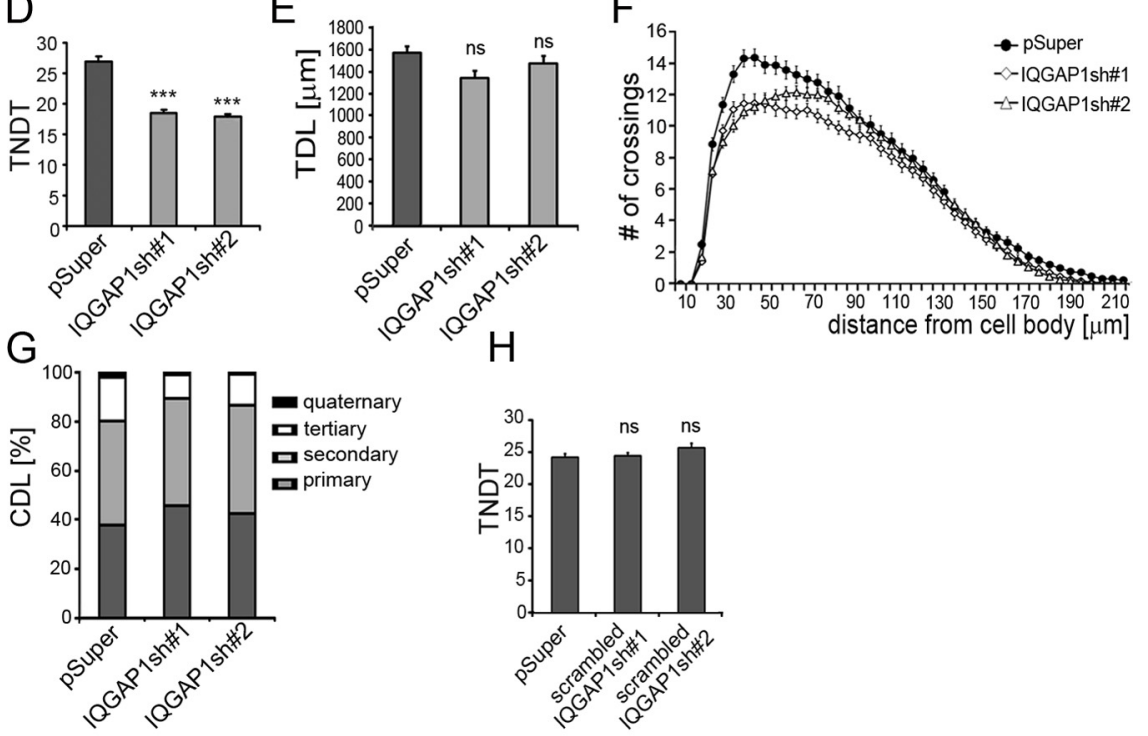

Figure 9. Effects of IQGAP1 knockdown on dendritic arbor morphology of hippocampal neurons. $A$, Representative images of RAT-2 cells transfected with IQGAP1sh\#2 cloned into pSuper ${ }^{\text {GFP }}$ for $2 \mathrm{~d}$ and immunostained for endogenous IQGAP1. Scale bar, $20 \mu \mathrm{m}$. B, Average immunofluorescence intensity of IQGAP1 in RAT-2 cells after transfection with IQGAP1 shRNAs. Bars indicate SEM ( ${ }^{* * *} p<0.001$; Kruskal-Wallis test). C, Representative images of neurons transfected at DIV7 for $5 \mathrm{~d}$ with pSuper vector or IQGAP1sh\#1 or \#2. GFP was cotransfected to identify transfected cells and to visualize neuronal morphology. Scale bar, $20 \mu \mathrm{m}$. $\boldsymbol{D}-\boldsymbol{G}$, TNDT (D), TDL $(\boldsymbol{E})$, Sholl analysis $(\boldsymbol{F})$, and CDL (G) of hippocampal neurons after IQGAP1 knockdown. $\boldsymbol{H}$, TNDT of hippocampal neurons transfected at DIV7 for $5 \mathrm{~d}$ with pSuper or scrambled IQGAP1sh\#1 or \#2. Error bars indicate SEM $\left({ }^{* * *} p<0.001\right.$; Kruskal-

\section{Actin polymerization rescues CLIP-170 and IQGAP1 knockdown phenotype}

The interaction between CLIP-170 and IQGAP1 may regulate the cross talk between microtubules and the actin cytoskeleton (Fukata et al., 2002). To investigate the potential relationship between CLIP-170 and actin cytoskeleton stabilization in dendrite morphogenesis, we first tested whether the effects of CLIP-170 depletion on dendritic arborization can be reversed by 
A
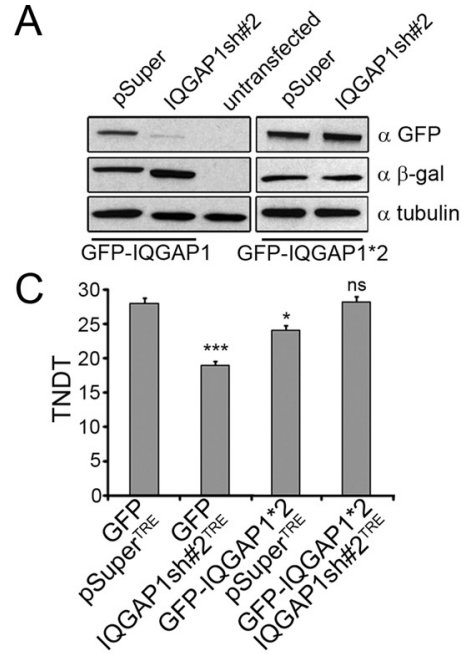

B
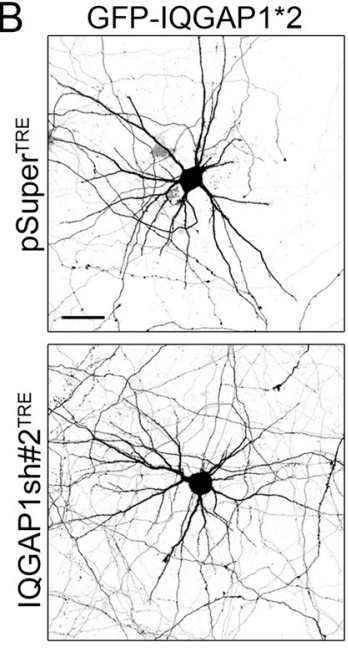
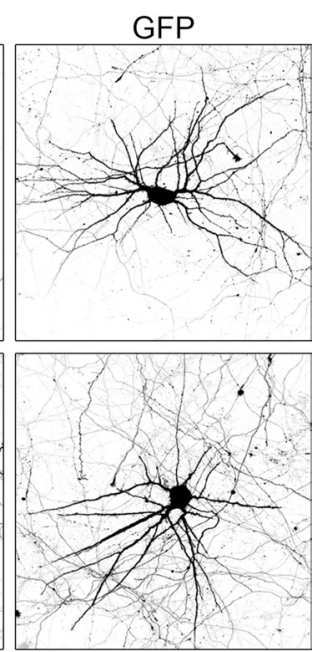

Figure 10. IQGAP1*2 overexpression in IQGAP1 knockdown cells results in improvement of dendritic tree morphology. $\boldsymbol{A}$, Assessment of GFP-IQGAP1*2 resistance to IQGAP1sh\#2 in COS7 cotransfected with wild-type (GFP-IQGAP1) or shRNA\#2-resistant IQGAP1 (GFP-IQGAP1*2) and pSuper or IQGAP1sh\#2 for 48 h. B, Representative images of hippocampal neurons cotransfected at DIV7 with either GFP-IQGAP1*2 or GFP and control pSuper ${ }^{\text {TRE }}$ vector or IQGAP1sh\#2 ${ }^{\text {TRE }}$, treated with doxycycline $(1 \mu \mathrm{g} / \mathrm{ml})$ the next day, and fixed $6 \mathrm{~d}$ after transfection. Neuronal morphology was visualized by cotransfected mRFP. Scale bar, $20 \mu \mathrm{m}$. C, TNDT. Error bars indicate SEM $\left({ }^{* * *} p<0.001 ;{ }^{*} p<0.05\right.$; Kruskal-Wallis test).

jasplakinolide-forced F-actin stabilization. DIV7 neurons were transfected with CLIP-170sh\#2 ${ }^{\mathrm{TRE}}$. Twenty-four hours later, doxycycline and jasplakinolide were added to the medium for $5 \mathrm{~d}$. Indeed, addition of jasplakinolide prevented the decrease in TNDT induced by CLIP-170 knockdown (Fig. 12A,B). If IQGAP1 is a link between CLIP-170 and the actin cytoskeleton needed for proper dendritic morphology, then one could expect that its depletion should result in actin-dependent deterioration of dendritic arborization. Indeed, similar to the effects of CLIP170 depletion, the decrease in TNDT caused by IQGAP1 knockdown was also reduced by jasplakinolide treatment (Fig. 12C).

To further investigate the relationship between CLIP-170 and actin cytoskeleton in neurons, we performed the F-actin pulldown from rat hippocampal protein extracts, and we observed binding of CLIP-170 to actin filaments. However, when extracts were incubated with $100 \mathrm{nM}$ rapamycin or immunodepleted of IQGAP1, this interaction was greatly abolished (Fig. 12D). Thus we concluded that CLIP-170 and its interacting partner IQGAP1 exert their effects on dendrites via the actin cytoskeleton and $\mathrm{mTOR}$ serves as a regulator of this process. Altogether, the present data indicate microtubule-actin cytoskeleton cross talk as a mechanism of mTOR-driven dendritic growth.

\section{Discussion}

The present study describes a novel mechanism of control of dendritic arbor morphology in mammalian neurons via a +TIP family member-CLIP-170. This mechanism likely involves an mTOR-dependent interaction between CLIP-170 and IQGAP1, an actin-regulating protein that coordinates cross talk between dynamic microtubule plus-ends and the actin cytoskeleton. Interactions of CLIP-170 with IQGAP1 and subsequently F-actin are decreased by mTOR inhibition in neuronal cells. Both CLIP170 and IQGAP1, similarly to mTOR, are indispensible for proper dendritic arbor morphology, and dendritic arbor deterioration caused by their knockdown can be rescued by F-actin stabilization (jasplakinolide treatment). Moreover, the presence of CLIP-170 and IQGAP1 and microtubule dynamics are needed to support mTOR-dependent, PI3K-induced increases in den- dritic arbor complexity in rat hippocampal neurons. These observations suggest that in addition to previously described mTOR-dependent translational control of dendritogenesis (Jaworski et al., 2005), this kinase supports this process by enhancing interactions between microtubule and actin-binding proteins. This, in turn, can lead to anchoring and stabilization of microtubules or to microtubule-induced changes in actin dynamics needed for dendritic growth or stabilization.

\section{+TIPs regulate dendritic arbor morphology}

In different model organisms, proper dendritic arbor morphology depends on proteins to ensure proper regulation of microtubule polymerization (CRMP3, Cypin), stabilization (MAP1, MAP2, doublecortin), severing (spastin), and depolymerization (SCLIP) [for review, see Georges et al. (2008), Urbanska et al. (2008), Conde and Cáceres (2009), and Poulain and Sobel (2010)]. These observations strongly suggest the importance of microtubule dynamics for dendritic tree morphology. Therefore, it is surprising how little is known about the role of + TIPs that are crucial for microtubule dynamics during dendritic arborization. This is in contrast to numerous reports describing the contribution of + TIPs to neuronal polarization or axonal growth (Lee et al., 2004; Zhou et al., 2004; Jiménez-Mateos et al., 2005; Grabham et al., 2007). Additionally, we recently proved the importance of + TIPs for dendritic spine morphology (Jaworski et al., 2009). The present study provides evidence that knockdown of CLIP-170, a founding member of the + TIP protein family, is vital for several aspects of dendritic arbor morphology.

Ample evidence supporting our conclusion that + TIPs are important for dendritic growth derives from studies in Drosophila. At least three proteins, DLIS-1, Dhc64, and shortstop (also known as kakapo), respective homologs of Lis1, dynein heavy chain, and ACF7, were shown to regulate dendritic arborization in fruit flies (Prokop et al., 1998; Gao et al., 1999; Liu et al., 2000; Satoh et al., 2008). The question remains, however, how +TIPs contribute to dendritic growth. Satoh et al. (2008) postulated that dynein-dependent transport of "branching machinery" from the cell soma toward distal dendrite regions is crucial for proper patterning of dendritic arborization ( $d a)$ neurons. Indeed, in proximal dendrites of Drosophila and in mammalian neurons, the majority of microtubules have their plus-ends oriented toward the cell soma (Stepanova et al., 2003; Satoh et al., 2008), and dynein transport is a major route of cargo sorting into dendrites (Kapitein et al., 2010). The identity of this machinery remains largely unknown, but Rab5 endosomes have emerged as a component (Satoh et al., 2008). + TIPs can also contribute to dendrite growth in other ways. All three + TIPs (Lis1, dynein, and ACF7) can directly or indirectly link microtubules to the cell cortex or actin cytoskeleton (Kodama et al., 2003; Lansbergen and Akhmanova, 2006), another important factor for dendritic patterning (Urbanska et al., 2008). Whether these particular proteins can regulate dendritic arborization by such cross-linking remains 
to be established, but the work presented herein strongly supports such a scenario for CLIP-170.

\section{CLIP-170 and IQGAP1 cooperate to} establish proper dendritic morphology In principle, each of the proposed functions for CLIP-170 (i.e., regulation of microtubule dynamics, facilitation of dynein-dependent transport, and crosslinking microtubules to the actin cytoskeleton) may importantly contribute to CLIP-170's effects on proper dendritic morphology. However, neither $\mathrm{N}$ - nor C-terminal deletion mutants could fully rescue the CLIP-170 knockdown phenotype. Protein interactions of the $\mathrm{N}$ terminus supporting net microtubule growth could potentially allow the advancement of microtubules into a newly formed filopodium and help branch formation. CLIP-170 also interacts via its $\mathrm{C}$ terminus with $\mathrm{p} 150^{\text {Glued }}$, a part of the dynein-dynactin complex, and Lis1, a role for which in dendrites was discussed above. The present study focused on cross-linking two types of cytoskeletons. Interactions between + TIPs and actin-regulating proteins have been shown to be a basis for morphological changes in neurons, including the formation of neurites (EB3 and drebrin) (Geraldo et al., 2008) and mature dendritic spines (EB3 and p140Cap) (Jaworski et al., 2009). We report that CLIP170 can interact with IQGAP1, and both proteins regulate dendritic arborization in an actin-dependent fashion.

IQGAP1 has been shown to transiently stabilize microtubules at the cell cortex of non-neuronal cells by interaction with CLIP-170 (Fukata et al., 2002; Watanabe et al., 2004). Based on these observations, CLIP-170 and IQGAP1 may coordinate dendritic growth simply by stabilizing microtubules at a site of a new dendritic branch formation. This would transiently polarize and enhance transport along microtubules toward future dendrites. A transportonly mechanism does not explain, however, why jasplakinolide treatment prevents dendritic arbor deterioration caused by either CLIP-170 or IQGAP1 knockdown. This observation suggests that in addition to microtubule stabilization at the cell cortex, CLIP170-IQGAP1-dependent dendritic growth also involves interdependent changes in actin cytoskeleton. Indeed, the CLIP-170 interaction with IQGAP1 can also take part in actin regulation. First, in vitro IQGAP1 stimulates actin polymerization as well as actin cross-linking (Briggs and Sacks, 2003). Second, Kholmanskikh et al. (2006) showed that in granular cerebellar and neonatal hippocampal neurons, CLIP-170 and IQGAP1 are in a protein complex that regulates F-actin at a leading edge of migrating neurons, in growing neurites, and somal plasma membrane. In addition, in this work we show that (1) CLIP-170 requires IQGAP1 and mTOR to bind to F-actin and (2) negative effects of CLIP-170 and IQGAP1 knockdown on dendritic arborization are interdependent and can be rescued by actin stabilization, forced
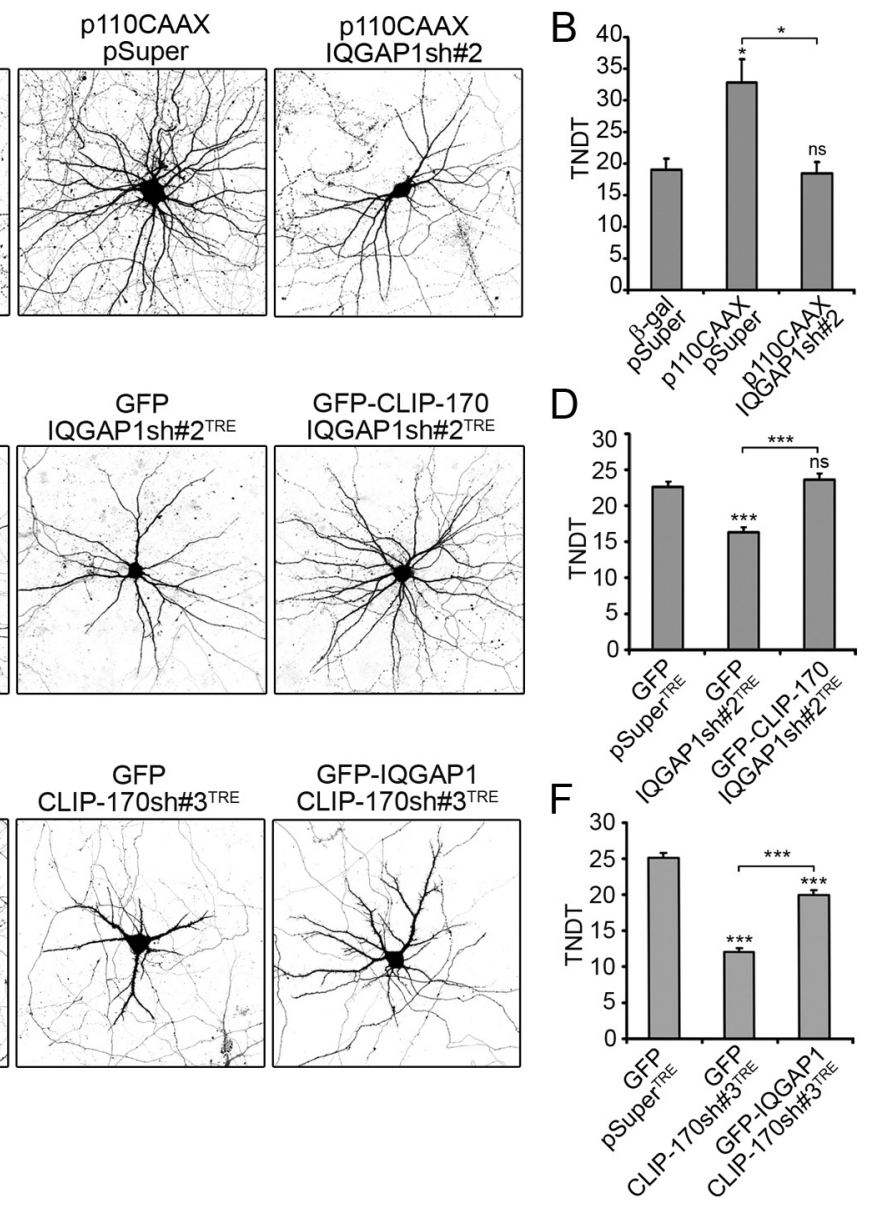

Figure 11. Knockdown of IQGAP1 decreases dendritic branching of wild-type and PI3K-transfected hippocampal neurons and can be rescued by CLIP-170. A, Representative images of hippocampal neurons cotransfected at DIV7 with IQGAP1sh\#2 or pSuper control vector together with $\beta$-galactosidase or p110CAAX for $5 \mathrm{~d}$. GFP was cotransfected for cell morphology visualization. Scale , $20 \mu \mathrm{m}$. B , TNDT of hippocampal neurons after transfection. C, Representative images of neurons transfected at DIV7 for $5 \mathrm{~d}$ neuronal morphology. Scale bar, $20 \mu \mathrm{m}$. D TNDT of hippocampal neurons transfected with IQGAP1sh\#2 alone or with GFP-CLIP170. E, Neurons after transfection at DIV7 and 5 dexpression of CLIP-170sh\#3 or pSuper together with GFP or GFP-IQGAP1. GFP was cotransfected to identify transfected cells and to visualize neuronal morphology. Scale bar, $20 \mu \mathrm{m}$. $\boldsymbol{F}$, TNDT of hippocampal neurons after transfection. Error bars indicate SEM $\left({ }^{* * *} p<0.001,{ }^{*} p<0.05\right.$; Kruskal-Wallis test).

by jasplakinolide. Taking all the above into consideration, although we cannot rule out alternative scenarios, in which CLIP170 and IQGAP1 may regulate F-actin independent of each other (Lewkowicz et al., 2008), we postulate that formation of a CLIP170-IQGAP1 complex could be involved in shaping dendritic arbors by mTOR, transiently stabilizing and cross-linking microtubules with the cellular cortex, and regulating F-actin (Fig. 12 E).

\section{mTOR contributes to dendritic arbor shape by several} mechanisms

In our previous work, we showed that mTOR-dependent dendritic arborization requires protein synthesis (Jaworski et al., 2005). mTOR kinase is best known for its key role in the control of translation, and axon formation, for example, has been shown to be regulated by mTOR-driven translation of microtubuleregulating proteins such as SAD kinases (Choi et al., 2008) or CRMP-2 (Morita and Sobue, 2009). Although newly translated proteins important for mTOR-dependent dendritic growth have not yet been identified, several candidates are among those proteins locally translated in dendrites in response to brain-derived neurotrophic factor (BDNF) or insulin (Schratt et al., 2004; Lee et 
A
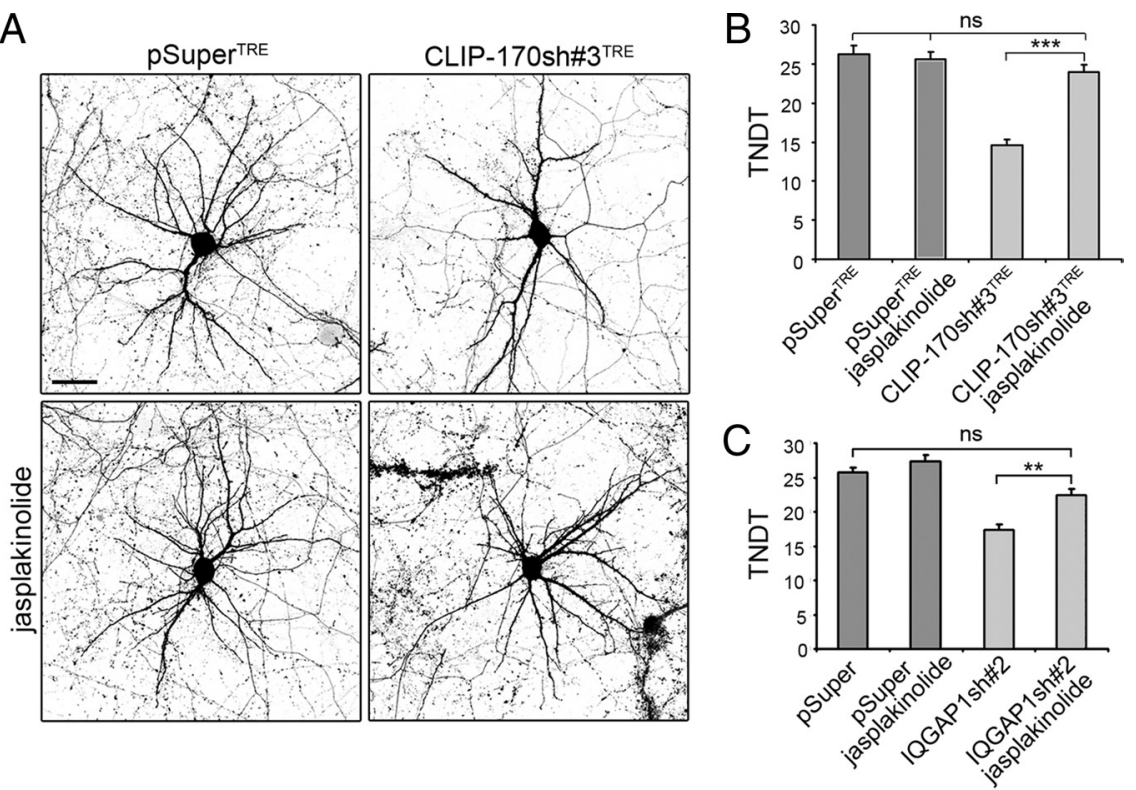

$\mathrm{D}$
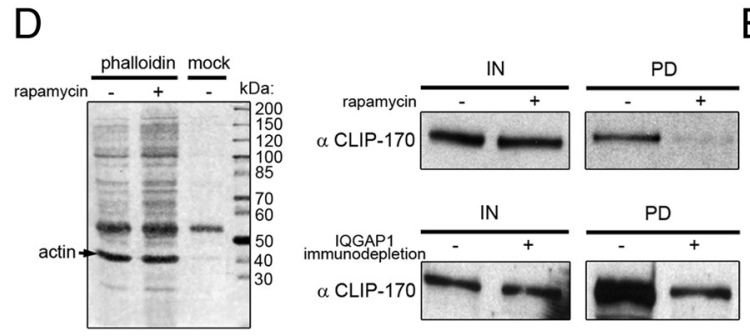

$\mathrm{E}$

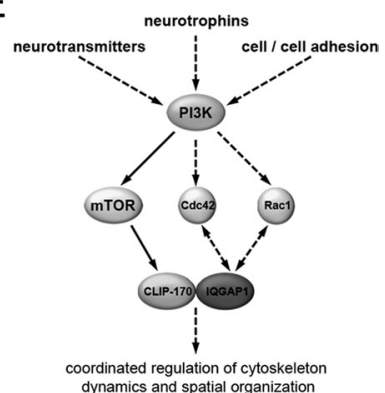

Figure 12. Forced actin stabilization prevents effects of CLIP-170 or IQGAP1 depletion in developing hippocampal neurons. $A$, Representative images of hippocampal neurons cotransfected at DIV7 with pSuper ${ }^{\text {TRE }}$ vector or CLIP-170sh\#3 ${ }^{\text {TRE }}$ treated with 1 $\mu \mathrm{g} / \mathrm{ml}$ doxycycline and $10 \mathrm{~nm}$ jasplakinolide $24 \mathrm{~h}$ later and fixed $6 \mathrm{~d}$ after transfection. Cells were cotransfected with GFP-encoding vector for morphology visualization. Scale bar, $20 \mu \mathrm{m}$. $\boldsymbol{B}$, Analysis of the TNDT of transfected neurons. C, TNDT of hippocampal neurons after IQGAP1 knockdown with sh\#2 and $4 \mathrm{~d}$ treatment with 10 nm jasplakinolide. Error bars indicate SEM (*** $p<0.001$, ${ }^{* *} p<0.01$; Kruskal-Wallis test). $\boldsymbol{D}$, Pull-down of F-actin from vehicle or rapamycin-treated (100 nM) rat hippocampal homogenates. Left, Silver-stained gel confirming presence of actin bound with biotinylated phalloidin. Right, Western blot analysis revealing rapamycin-sensitive and IQGAP1-dependent interaction of CLIP-170 with F-actin resin. $\boldsymbol{E}$, A proposed model of a novel mTOR-dependent mechanism of dendritic arbor development regulation.

al., 2005) (for detailed discussion, see also Swiech et al., 2008). However, the present study shows that, in addition to protein translation, PI3K-driven dendritic arborization, which is an mTOR-controlled process (Jaworski et al., 2005; Kumar et al., 2005), depends on microtubule dynamics and the presence of both CLIP-170 and IQGAP1. Moreover, we confirm that CLIP170 is an mTOR kinase substrate [as shown by Choi et al. (2002) in non-neuronal cells] and reveal mTOR-dependent regulation of CLIP-170 protein-protein interactions.

PI3K activates mTORC1 in response to several stimuli known to induce dendritic growth, such as neurotransmitters (e.g., glutamate), neurotrophins (e.g., BDNF), and the extracellular matrix (e.g., chicken acidic leucine-rich epidermal growth factorlike domain-containing brain protein/neuroglycan C) (Jaworski and Sheng, 2006; Brandt et al., 2007; Swiech et al., 2008; Urbanska et al., 2008). However, active PI3K also induces small GTPases such as Rac1 and Cdc42 that interact with IQGAP1. Thus, we speculate that activation of mTOR by extracellular signals may allow the formation of the IQGAP1-CLIP-170 complex with PI3K-activated Cdc42 or Rac1 to converge mTOR and small Rho
GTPase signaling pathways downstream of PI3K to coordinate the regulation of microtubules and actin during dendritic growth (Fig. 12E). The present study adds the control of microtubule-actin interactions to the repertoire of mTOR during dendritic arbor growth.

\section{References}

Akhmanova A, Hoogenraad CC (2005) Microtubule plus-end-tracking proteins: mechanisms and functions. Curr Opin Cell Biol 17:47-54.

Banker G, Goslin K (1988) Developments in neuronal cell culture. Nature 336:185-186.

Brandt N, Franke K, Rasin MR, Baumgart J, Vogt J, Khrulev S, Hassel B, Pohl EE, Sestan N, Nitsch R, Schumacher S (2007) The neural EGF family member CALEB/NGC mediates dendritic tree and spine complexity. EMBO J 26:2371-2386.

Briggs MW, Sacks DB (2003) IQGAP proteins are integral components of cytoskeletal regulation. EMBO Rep 4:571-574.

Brummelkamp TR, Bernards R, Agami R (2002) A system for stable expression of short interfering RNAs in mammalian cells. Science 296:550-553.

Choi JH, Bertram PG, Drenan R, Carvalho J, Zhou HH, Zheng XF (2002) The FKBP12rapamycin-associated protein (FRAP) is a CLIP-170 kinase. EMBO Rep 3:988-994.

Choi YJ, Di Nardo A, Kramvis I, Meikle L, Kwiatkowski DJ, Sahin M, He X (2008) Tuberous sclerosis complex proteins control axon formation. Genes Dev 22:2485-2495.

Conde C, Cáceres A (2009) Microtubule assembly, organization and dynamics in axons and dendrites. Nat Rev Neurosci 10:319-332.

Coquelle FM, Caspi M, Cordelières FP, Dompierre JP, Dujardin DL, Koifman C, Martin P, Hoogenraad CC, Akhmanova A, Galjart N, De Mey JR, Reiner O (2002) LIS1, CLIP-170's key to the dynein/dynactin pathway. Mol Cell Biol 22:3089-3102.

de Boer E, Rodriguez P, Bonte E, Krijgsveld J, Katsantoni E, Heck A, Grosveld F, Strouboulis J (2003) Efficient biotinylation and single-step purification of tagged transcription factors in mammalian cells and transgenic mice. Proc Natl Acad Sci U S A 100:7480-7485.

Fukata M, Watanabe T, Noritake J, Nakagawa M, Yamaga M, Kuroda S, Matsuura Y, Iwamatsu A, Perez F, Kaibuchi K (2002) Rac1 and Cdc42 capture microtubules through IQGAP1 and CLIP-170. Cell 109:873-885.

Galjart N (2005) CLIPs and CLASPs and cellular dynamics. Nat Rev Mol Cell Biol 6:487-498.

Gao FB, Brenman JE, Jan LY, Jan YN (1999) Genes regulating dendritic outgrowth, branching, and routing in Drosophila. Genes Dev 13:2549-2561.

Georges PC, Hadzimichalis NM, Sweet ES, Firestein BL (2008) The yinyang of dendrite morphology: unity of actin and microtubules. Mol Neurobiol 38:270-284.

Geraldo S, Khanzada UK, Parsons M, Chilton JK, Gordon-Weeks PR (2008) Targeting of the F-actin-binding protein drebrin by the microtubule plustip protein EB3 is required for neuritogenesis. Nat Cell Biol 10:1181-1189.

Grabham PW, Seale GE, Bennecib M, Goldberg DJ, Vallee RB (2007) Cytoplasmic dynein and LIS1 are required for microtubule advance during growth cone remodeling and fast axonal outgrowth. J Neurosci 27:5823-5834. 
Hoogenraad CC, Bradke F (2009) Control of neuronal polarity and plasticity - a renaissance for microtubules? Trends Cell Biol 19:669-676.

Hoogenraad CC, Akhmanova A, Grosveld F, De Zeeuw CI, Galjart N (2000) Functional analysis of CLIP-115 and its binding to microtubules. J Cell Sci 113:2285-2297.

Hoogenraad CC, Milstein AD, Ethell IM, Henkemeyer M, Sheng M (2005) GRIP1 controls dendrite morphogenesis by regulating EphB receptor trafficking. Nat Neurosci 8:906-915.

Hoogenraad CC, Feliu-Mojer MI, Spangler SA, Milstein AD, Dunah AW, Hung AY, Sheng M (2007) Liprinalphal degradation by calcium/ calmodulin-dependent protein kinase II regulates LAR receptor tyrosine phosphatase distribution and dendrite development. Dev Cell 12:587-602.

Jan YN, Jan LY (2010) Branching out: mechanisms of dendritic arborization. Nat Rev Neurosci 11:316-328.

Jaworski J, Sheng M (2006) The growing role of mTOR in neuronal development and plasticity. Mol Neurobiol 34:205-219.

Jaworski J, Spangler S, Seeburg DP, Hoogenraad CC, Sheng M (2005) Control of dendritic arborization by the phosphoinositide-3'-kinase-Aktmammalian target of rapamycin pathway. J Neurosci 25:11300-11312.

Jaworski J, Hoogenraad CC, Akhmanova A (2008) Microtubule plus-end tracking proteins in differentiated mammalian cells. Int J Biochem Cell Biol 40:619-637.

Jaworski J, Kapitein LC, Gouveia SM, Dortland BR, Wulf PS, Grigoriev I, Camera P, Spangler SA, Di Stefano P, Demmers J, Krugers H, Defilippi P, Akhmanova A, Hoogenraad CC (2009) Dynamic microtubules regulate dendritic spine morphology and synaptic plasticity. Neuron 61:85-100.

Jiménez-Mateos EM, Paglini G, González-Billault C, Cáceres A, Avila J (2005) End binding protein-1 (EB1) complements microtubuleassociated protein-1B during axonogenesis. J Neurosci Res 80:350-359.

Kapitein LC, Schlager MA, Kuijpers M, Wulf PS, van Spronsen M, MacKintosh FC, Hoogenraad CC (2010) Mixed microtubules steer dyneindriven cargo transport into dendrites. Curr Biol 20:290-299.

Kholmanskikh SS, Koeller HB, Wynshaw-Boris A, Gomez T, Letourneau PC, Ross ME (2006) Calcium-dependent interaction of Lis1 with IQGAP1 and Cdc42 promotes neuronal motility. Nat Neurosci 9:50-57.

Kim DH, Sarbassov DD, Ali SM, King JE, Latek RR, Erdjument-Bromage H, Tempst P, Sabatini DM (2002) mTOR interacts with raptor to form a nutrient-sensitive complex that signals to the cell growth machinery. Cell 110:163-175.

Kodama A, Karakesisoglou I, Wong E, Vaezi A, Fuchs E (2003) ACF7: an essential integrator of microtubule dynamics. Cell 115:343-354.

Komarova YA, Akhmanova AS, Kojima S, Galjart N, Borisy GG (2002) Cytoplasmic linker proteins promote microtubule rescue in vivo. J Cell Biol 159:589-599.

Konopka W, Duniec K, Mioduszewska B, Proszynski T, Jaworski J, Kaczmarek L (2005) hCMV and Tet promoters for inducible gene expression in rat neurons in vitro and in vivo. Neurobiol Dis 19:283-292.

Kumar V, Zhang MX, Swank MW, Kunz J, Wu GY (2005) Regulation of dendritic morphogenesis by Ras-PI3K-Akt-mTOR and Ras-MAPK signaling pathways. J Neurosci 25:11288-11299.

Lansbergen G, Akhmanova A (2006) Microtubule plus end: a hub of cellular activities. Traffic 7:499-507.

Lansbergen G, Komarova Y, Modesti M, Wyman C, Hoogenraad CC, Goodson HV, Lemaitre RP, Drechsel DN, van Munster E, Gadella TW Jr, Grosveld F, Galjart N, Borisy GG, Akhmanova A (2004) Conformational changes in CLIP-170 regulate its binding to microtubules and dynactin localization. J Cell Biol 166:1003-1014.

Lee CC, Huang CC, Wu MY, Hsu KS (2005) Insulin stimulates postsynaptic density-95 protein translation via the phosphoinositide 3-kinase-Aktmammalian target of rapamycin signaling pathway. J Biol Chem 280:18543-18550.

Lee H, Engel U, Rusch J, Scherrer S, Sheard K, Van Vactor D (2004) The microtubule plus end tracking protein Orbit/MAST/CLASP acts downstream of the tyrosine kinase $\mathrm{Abl}$ in mediating axon guidance. Neuron 42:913-926.

Lewkowicz E, Herit F, Le Clainche C, Bourdoncle P, Perez F, Niedergang F (2008) The microtubule-binding protein CLIP-170 coordinates mDial and actin reorganization during CR3-mediated phagocytosis. J Cell Biol 183:1287-1298.

Liu Z, Steward R, Luo L (2000) Drosophila Lis1 is required for neuroblast proliferation, dendritic elaboration and axonal transport. Nat Cell Biol 2:776-783.

Meijering E, Jacob M, Sarria JC, Steiner P, Hirling H, Unser M (2004) Design and validation of a tool for neurite tracing and analysis in fluorescence microscopy images. Cytometry A 58:167-176.

Morita T, Sobue K (2009) Specification of neuronal polarity regulated by local translation of CRMP2 and tau via the mTOR-p70S6K pathway. J Biol Chem 284:27734-27745.

Perez F, Diamantopoulos GS, Stalder R, Kreis TE (1999) CLIP-170 highlights growing microtubule ends in vivo. Cell 96:517-527.

Pierre P, Pepperkok R, Kreis TE (1994) Molecular characterization of two functional domains of CLIP-170 in vivo. J Cell Sci 107:1909-1920.

Poulain FE, Sobel A (2010) The microtubule network and neuronal morphogenesis: dynamic and coordinated orchestration through multiple players. Mol Cell Neurosci 43:15-32.

Prokop A, Uhler J, Roote J, Bate M (1998) The kakapo mutation affects terminal arborization and central dendritic sprouting of Drosophila motorneurons. J Cell Biol 143:1283-1294.

Ren JG, Li Z, Crimmins DL, Sacks DB (2005) Self-association of IQGAP1: characterization and functional sequelae. J Biol Chem 280:34548-34557.

Rickard JE, Kreis TE (1990) Identification of a novel nucleotide-sensitive microtubule-binding protein in HeLa cells. J Cell Biol 110:1623-1633.

Rickard JE, Kreis TE (1991) Binding of pp170 to microtubules is regulated by phosphorylation. J Biol Chem 266:17597-17605.

Satoh D, Sato D, Tsuyama T, Saito M, Ohkura H, Rolls MM, Ishikawa F, Uemura T (2008) Spatial control of branching within dendritic arbors by dynein-dependent transport of Rab5-endosomes. Nat Cell Biol 10:1164-1171.

Schratt GM, Nigh EA, Chen WG, Hu L, Greenberg ME (2004) BDNF regulates the translation of a select group of mRNAs by a mammalian target of rapamycin-phosphatidylinositol 3-kinase-dependent pathway during neuronal development. J Neurosci 24:7366-7377.

Schuyler SC, Pellman D (2001) Microtubule "plus-end-tracking proteins": the end is just the beginning. Cell 105:421-424.

Sholl DA (1953) Dendritic organization in the neurons of the visual and motor cortices of the cat. J Anat 87:387-406.

Siegrist SE, Doe CQ (2007) Microtubule-induced cortical cell polarity. Genes Dev 21:483-496.

Steinmetz MO, Akhmanova A (2008) Capturing protein tails by CAP-Gly domains. Trends Biochem Sci 33:535-545.

Stepanova T, Slemmer J, Hoogenraad CC, Lansbergen G, Dortland B, De Zeeuw CI, Grosveld F, van Cappellen G, Akhmanova A, Galjart N (2003) Visualization of microtubule growth in cultured neurons via the use of EB3-GFP (end-binding protein 3-green fluorescent protein). J Neurosci 23:2655-2664.

Stoppini L, Buchs PA, Muller D (1991) A simple method for organotypic cultures of nervous tissue. J Neurosci Methods 37:173-182.

Swiech L, Perycz M, Malik A, Jaworski J (2008) Role of mTOR in physiology and pathology of the nervous system. Biochim Biophys Acta 1784:116-132.

Urbanska M, Blazejczyk M, Jaworski J (2008) Molecular basis of dendritic arborization. Acta Neurobiol Exp (Wars) 68:264-288.

van de Wetering M, Oving I, Muncan V, Pon Fong MT, Brantjes H, van Leenen D, Holstege FC, Brummelkamp TR, Agami R, Clevers H (2003) Specific inhibition of gene expression using a stably integrated, inducible small-interfering-RNA vector. EMBO Rep 4:609-615.

Watanabe T, Wang S, Noritake J, Sato K, Fukata M, Takefuji M, Nakagawa M, Izumi N, Akiyama T, Kaibuchi K (2004) Interaction with IQGAP1 links APC to Rac1, Cdc42, and actin filaments during cell polarization and migration. Dev Cell 7:871-883.

Witte H, Neukirchen D, Bradke F (2008) Microtubule stabilization specifies initial neuronal polarization. J Cell Biol 180:619-632.

Zhou FQ, Zhou J, Dedhar S, Wu YH, Snider WD (2004) NGF-induced axon growth is mediated by localized inactivation of GSK-3beta and functions of the microtubule plus end binding protein APC. Neuron 42:897-912. 